\title{
Reciprocal impacts of telomerase activity and tumor cell differentiation in neuroblastoma tumor biology
}

Eun Young Yu ${ }^{1}$, Syed S. Zahid ${ }^{1}$, Sarah Aloe ${ }^{1}$, Erik Falck-Pedersen ${ }^{1}$, Xi Kathy Zhou $^{2}$, Nai-Kong V. Cheung ${ }^{3}$, and Neal F. $\operatorname{Lue}^{1,4 *}$

${ }^{1}$ Department of Microbiology \& Immunology, W. R. Hearst Microbiology Research Center, Weill Cornell Medicine, New York, NY, USA

${ }^{2}$ Department of Population Health Sciences, Weill Cornell Medicine, New York, NY, USA

${ }^{3}$ Department of Pediatrics, Memorial Sloan Kettering Cancer Center, New York, NY, USA

${ }^{4}$ Sandra and Edward Meyer Cancer Center, Weill Cornell Medical College, New York, NY, USA

Correspondence:

Neal F. Lue

Department of Microbiology and Immunology

Weill Cornell Medicine

1300 York Avenue

New York, NY 10065

Tel: +1212 746-6506

Fax: $+1212746-8587$

Email: nflue@med.cornell.edu

$\underline{\text { Running title: }}$ Telomere, cell lineage, and immune response in neuroblastoma

Key Words: neuroblastoma, telomere, telomerase, cell lineage, adrenergic, mesenchymal, DNA sensing, innate immunity 


\begin{abstract}
Telomere maintenance and tumor cell differentiation have been separately implicated in neuroblastoma malignancy. Their mechanistic connection is unclear. We analyzed neuroblastoma cell lines and morphologic subclones representing the adrenergic (ADRN) and mesenchymal (MES) differentiation states and uncovered sharp differences in their telomere protein and telomerase activity levels. Pharmacologic conversion of ADRN into MES cells elicited consistent and robust changes in the expression of telomere-related proteins. Conversely, stringent down-regulation of telomerase activity triggers the differentiation of ADRN into MES cells, which was reversible upon telomerase upregulation. Interestingly, the MES differentiation state is associated with elevated levels of innate immunity factors, including key components of the DNA-sensing pathway. Accordingly, MES but not ADRN cells can mount a robust response to viral infections in vitro. A gene expression signature based on telomere and cell lineage-related factors can cluster neuroblastoma tumor samples into predominantly ADRN or MES-like groups, with distinct clinical outcomes. Our findings establish a novel mechanistic connection between telomere and differentiation and suggest that manipulating telomeres may suppress malignancy not only by limiting the tumor growth potential but also by inducing tumor cell differentiation and altering its immunogenicity.
\end{abstract}

\title{
Key Words
}

neuroblastoma, telomere, telomerase, cell lineage, adrenergic, mesenchymal, DNA sensing, innate immunity 


\section{Introduction}

Neuroblastoma (NB), a complex childhood cancer, has a unique biology of spontaneous remission and lethal progression depending on its genetic makeup 1,2. Arising from pluripotent neural crest precursors of the sympathoadrenal lineage ${ }^{3,4}$, NB has been known to manifest phenotypic diversity that mirrors the developmental potential of neural crest progenitor cells. In early studies of cell lines with distinct morphologies and biochemical markers, tumor cells were classified as N (neuroblastic), I (intermediate), and S (substrate-adherent) cells, and these cell types were shown to be interconvertible via pharmacologic treatment ${ }^{5,6,7,8}$. The different cell types were also found to exhibit varying degrees of tumorigenicity in animal models; the I cells, which manifest more features of stem cells, were particularly malignant ${ }^{6}$. More recently, detailed transcription and epigenetic profiling has reclassified NB tumors into the adrenergic (ADRN) and mesenchymal (MES) lineages that are defined by the activation of distinct super enhancers $9,10,11$. Inspection of the phenotypic features associated with the earlier morphologic/biochemical and the more recent genetic classifications suggests broad congruence between the two schemes, with the $\mathrm{N}$ and I cells showing substantial similarities to the ADRN lineage and the S cells resembling MES. For example, both S and MES cells harbor high levels of cytoskeletal proteins associated with the mesenchymal phenotype. Indeed, one pair of cell lines is shared between the earlier and more recent studies and their designations support the N/ADRN and S/MES groupings ${ }^{5,10}$. (For consistency, the ADRN and MES designations are used throughout this study for cell lines that were initially classified according to the N/I/S classification scheme.) Notably, multiple studies implicate the state of tumor cell differentiation in disease progression and treatment response. For example, the MES cells are more chemo-resistant and may be enriched in relapse and in metastatic diseases ${ }^{10,12,13 .}$ In contrast, the ADRN cells tend to predominate at diagnosis and show greater sensitivity to chemotherapies ${ }^{10,11 .}$

Another facet of NB biology that has been strongly implicated in disease progression is telomere maintenance ${ }^{14,15}$. Telomeres are dynamic nucleoprotein structures that stabilize the tips of chromosomes against aberrant DNA damage response and DNA repair ${ }^{16}$. As such, telomeres are crucial for both normal and cancer cell proliferation ${ }^{17}$. However, owing to incomplete end replication, telomere DNAs (a repetitive 6-base sequence) are subject to progressive loss with successive cell division and must be replenished to maintain an adequate reserve for cell 
proliferation. Indeed, recent studies of extensive tumor collections have confirmed a strong association between the presence of telomere maintenance mechanisms (TMMs) and high-risk disease as well as poor prognosis for NB 18, 19. The predominant TMM in NB is a special reverse transcriptase named telomerase, which uses the catalytic TERT protein in conjunction with an RNA template to add telomere repeats ${ }^{20,21}$. The second major TMM, known as $\mathrm{ALT}$ (alternative lengthening of telomeres), is a recombination pathway that resembles break-induced replication ${ }^{22}$, 23. Consistent with the critical importance of TMMs, three of the most frequent genetic alterations in high-risk NB (MYCN amplification, TERT promoter rearrangement, and ATRX inactivation) are mutually exclusive and are each strongly linked to TMM activation - specifically, MYCN and TERT mutations give rise to high telomerase expression and ATRX loss triggers the ALT pathway ${ }^{14,15,24}$. The special importance of TMMs in NB may be a consequence of "telomere trimming" 25, 26, a telomere shortening pathway that we recently demonstrated in a substantial fraction of high-risk NB tumors ${ }^{27}$. The need to compensate for the telomere loss precipitated by both incomplete end replication and telomere trimming may render NB especially dependent on TMMs. Besides telomerase and ALTrelated factors, many other proteins are known to regulate telomere length homeostasis and telomere protection. For example, the six-protein assembly (RAP1-TRF2-TIN2-TRF1-TPP1-POT1) that coats telomeres, known as shelterin, is crucial for suppressing aberrant DNA repair and DNA damage response at chromosome ends ${ }^{16,28}$. Although their roles in NB have not been investigated, dys-regulation of the shelterin proteins will probably impact tumor growth in line with observations for other cancers. There is thus growing interest in developing telomere and TERT-directed therapies against NB ${ }^{29,30}$.

Remarkably, multiple studies have implicated telomere-related factors in neural development and neural differentiation. For example, the shelterin component TRF2 has been reported to regulate neural cell differentiation and neural protection, and these functions are at least partly mediated through non-telomeric pathways ${ }^{31,32,33 .}$ More specifically, a short, cytoplasmic isoform of TRF2 (TRF2-S) is thought to antagonize the silencing effect of REST to promote neural differentiation ${ }^{31}$. Thus, telomere proteins have the potential to alter the differentiation of neural crest-derived NB tumors in accordance with the developmental origin of these cells ${ }^{3,4}$. Likewise, telomerase and its catalytic protein subunit TERT may have similar potentials in NB differentiation given their proposed functions in 
neural development ${ }^{34,35}$. However, little is known about the roles of these telomere maintenance and protection factors in the ADRN and MES lineages of NB tumor cells.

In this study, we set out to address the roles of telomere-related factors in the different lineages of NB tumor cells. Using isogenic cells lines that display either ADRN or MES phenotypes, we showed that the levels of telomere factors vary dramatically, with the ADRN cells generally harboring much higher levels of such factors. Pharmacologically converting ADRN cells into MES cells triggered the expected changes in telomere protein profiles. Conversely, inhibiting telomerase activity in ADRN cells induced their conversion into MES cells in a reversible manner. Together, these findings establish a tight mechanistic connection between telomere regulation and NB lineage conversion. Another notable finding was the strong up-regulation of DNA-sensing and innate immunity factors in MES cells, which supports several recent reports of similar associations ${ }^{36,37}$. By linking telomere regulation to tumor cell differentiation and immunogenicity in NB, our study provides potential explanations for the disparate clinical outcomes of NB cases, and reinforces the rationale for developing telomere-directed therapies against this aggressive pediatric cancer.

\section{Results}

\section{$\underline{A D R N}$ and MES cells are distinguished by prominent differences in telomere proteins}

Three matched pairs of ADRN and MES cell lines (LA1-55N [ADRN] and LA1-5S [MES]; LA1-66N [ADRN] and LA1-6S [MES]; SH-SY5Y [ADRN] and SH-EP1 [MES]) were analyzed with respect to the levels of previously defined lineage markers (e.g., SLUG and HES1 for MES and GATA3 for ADRN) as well as telomere-related proteins (Fig. 1a and 1b). As expected, the different cell lines preferentially express the associated differentiation markers (Fig. 1a). Also similar to previous reports, the levels of MYCN are high in ADRN cells harboring MYCN amplification ${ }^{6}$. Notably, multiple telomere-related factors were found to be differentially expressed in ADRN and MES cells (Fig. 1b). These include three shelterin components that were over-expressed in ADRN cells relative to MES (TRF1, TRF2 and TPP1), and another component that was elevated in MES (POT1). Also differentially expressed are other factors implicated in telomere or chromosome maintenance. In general, proteins involved telomere DNA synthesis or repair (ATRX, 
RIF1, RPA70, RTEL1, STN1) tend to be more abundant in ADRN cells. However, two subunits of the primase-Pol $\alpha$ complex (PRIM1 and POLA2), which are important for telomere replication as well as telomere C-strand extension 38, 39 , are more abundant in MES cells. These results suggest that the telomere nucleoprotein structure may undergo remodeling during tumor cell differentiation between ADRN and MES cells.

To determine if changes in telomere protein levels are associated with changes in telomere nucleoprotein configuration, we analyzed the cellular distribution of TRF1 and TRF2, the two major double-strand telomere binding proteins in the shelterin complex (Fig. 1c and 1d, Supp. Fig. 1a). Consistent with their overall protein levels in Western analysis, both TRF1 and TRF2 displayed stronger signals and brighter foci in the ADRN cell line LA1-55N. As expected, TRF1 was exclusively in the nucleus and exhibited significant co-localization with telomere foci, especially in LA1-55N. In contrast, TRF2 displayed prominent non-nuclear staining in a cell type-specific manner. In LA1-55N, TRF2 was present in both the nucleus and the cytoplasm, and showed especially bright cytoplasmic foci that cluster in the peri-nuclear region (Fig. 1d and Supp. Fig. 1a). Moreover, the nuclear signals of TRF2 were predominantly nontelomeric, suggesting that the telomere chromatin of ADRN cells contain low levels of TRF2 despite the overall abundance of this protein (see also results for another ADRN cell line BE(2)N later). In LA1-5S, TRF2 showed faint nuclear staining that overlapped slightly with telomere foci. Parallel staining of TRF1 and TRF2 in HeLa cells revealed the expected nuclear localization and signal overlap with telomere foci (Fig. 1c and 1d), indicating that the unusual TRF2 pattern in ADRN cells is unlikely to be an artifact of staining. One possible explanation for the cytoplasmic TRF2 signal comes from earlier reports of TRF2-S, a short isoform found in the cytoplasm of neural cells ${ }^{31}$. In support of the presence of TRF2-S in NB, longer exposures of TRF2 Western blots revealed the presence of a 34 kDa immunereactive protein that is reminiscent of TRF2-S (Supp. Fig. 1b). Overall, the immunostaining supports the notion that there are lineage-specific differences between the telomere nucleoprotein structures of ADRN and MES cells.

The DNA-sensing pathway has been linked to telomere DNA damage and the ALT mechanism of telomere maintenance ${ }^{40,41}$. We analyzed several key components of this pathway in our cell lines, and observed dramatic upregulation of cGAS, STING and IRF3 in MES cells (Fig. 1a), suggesting that these cells may be more responsive to 
abnormal DNA stimuli. Other groups have recently reported differences in the immune profiles of ADRN and MES cells ${ }^{36,37}$.

Telomere lengths and telomerase activity differ between ADRN and MES cells, but exhibit no uniform pattern with respect to cell lineage

To determine if the differences in telomere proteins in ADRN and MES are associated with changes in telomere maintenance, we first analyzed telomere lengths in the respective cell lines using both standard Southern analysis and STELA, a PCR-based assay that preferentially detect short telomeres (Fig. $2 a-2 c)^{27}$. While each pair of cell lines showed some difference in the length distribution of telomeres, there was no consistent pattern with respect to cell lineage, even for matched ADRN and MES lines derived from the same tumor. Specifically, whereas LA1-55N (ADRN) harbored shorter telomeres than the matched LA1-5S (MES), the opposite was true for LA1-66N (ADRN) and LA1-6S (MES). We also analyzed single stranded DNA at telomeres, which reflect different aspects of telomere regulation; elevated C-strand is often due to "telomere trimming" 25, 26, 42 and elevated G-strand is a marker of telomere deprotection ${ }^{43}$. Significant differences between ADRN and MES were observed for C-strand ssDNA, but these differences were again not consistently related to the cell lineage (Fig. 2d). Finally, telomerase activities in the cell lines were assessed and were found to manifest MYCN-dependent cell lineage differences. In particular, telomerase activity was down regulated by 10-15 fold in matched MES cells, but only in MYCN-amplified tumor cell lines, i.e., in LA1-5S and LA1-6S but not in SH-EP1 (Fig. 2e). While tumor cell lineage does not strictly control telomere lengths or telomerase activity, the combined effects of telomere lengthening and shortening pathways on overall telomere lengths may impact cell growth. Among the lines derived from LAN-1, LA1-6S has the lowest telomerase activity and highest level of telomere trimming, which may explain its having the shortest telomeres and the slowest growth rate (Fig. 2c and Supp. Fig. 1c).

\section{Conversion of ADRN into MES cells is accompanied by telomere remodeling}

Chronic low dose BrdU treatment has been shown to effectively trigger the conversion of ADRN cells into MES cells

7,8. Indeed, when cultured in BrdU, the ADRN cell line BE(2)N (abbreviated from SK-N-BE(2)N) manifested progressive morphologic changes that culminated eventually in an MES-like state by day 26 (Fig. 3a). Consistent with gradual 
conversion to the MES lineage, telomerase activity in the cell population declined early and progressively to become nearly undetectable (> 100-fold reduced at day 26) (Fig. 3b). The levels of telomere factors, STING and MYCN likewise manifested the expected changes toward the MES expression profile (Fig. 3c). Localization TRF1 and TRF2 at early and late time points of BrdU treatment mirrored their distributions in ADRN and MES cells, respectively, with TRF2 showing peri-nuclear/cytoplasmic staining on day 4 and faint nuclear staining on day 19 (Supp. Fig. 2a and 2b). Interestingly, the decline in TRF1, TRF2 and MYCN levels occurred rapidly within the first 5 days, whereas the increase in STING was especially prominent during the late stage of conversion (after day 10 and continues beyond day 20). These findings corroborate the differences in telomere proteins and telomerase activity observed in naturally derived ADRN and MES cell lines, and support a progressive change in the gene expression program during BrdUinduced phenotypic conversion. Interestingly, the reduction in telomerase activity is not associated with significant changes in telomere lengths (Fig. 3d), echoing the inconsistent relationship between cell lineage and telomere lengths in naturally derived ADRN and MES cell lines.

To more comprehensively characterize gene expression changes during lineage conversion, we used RNA-seq to profile the transcriptomes of $\mathrm{BE}(2) \mathrm{N}$ at multiple time points during BrdU treatment. In support of the notion of gradual phenotypic conversion, we found that a progressively higher number of genes were up-regulated from early to late time points, and this applies to down-regulated genes as well (Fig. 3e). The heatmaps of individual genes further support progressive changes in the level of mRNAs (Fig. 3f). Comparison of the RNA-seq data to previously characterized signatures revealed a significant overlap between genes up-regulated by BrdU and the MES signature, as well as an overlap between genes down-regulated by BrdU and the ADRN signature (Fig. 3g). Moreover, pathway analysis of genes up-regulated in MES cells highlighted the involvement of cell adhesion/motility, epithelialmesenchymal transition, and immune regulatory genes in the phenotypic switch (Fig. 3h), further reinforcing the resemblance between naturally derived and BrdU-induced MES cells.

We tested the generality of our observations on BE(2)N by analyzing two other MYCN-amplified, ADRN-like cell lines: $\mathrm{BE}(2) \mathrm{C}$ (similar to $\mathrm{BE}(2) \mathrm{N}$ and thought to exhibit more stem cell characteristics) and SK-N-HM (derived from the brain metastasis of another $M Y C N$-amplified $N B$ case) ${ }^{7}$. Just like $B E(2) N$, BrdU-treated $B E(2) C$ displayed prominent 
changes in telomere, DNA-sensing, and lineage-related proteins (e.g., decreases in TRF1, ATRX, MYCN, and GATA3, as well as increases in PRIM1, POLA2, IRF3, STING, and SLUG) (Supp. Fig. 3a). Also resembling BE(2)N, while telomerase activity was greatly reduced by BrdU in $\mathrm{BE}(2) \mathrm{C}$, no significant change in telomere length was observed (Supp. Fig. 3b and 3c). These results mirror the lack of correlation between cell lineage and telomere lengths observed for naturally derived ADRN and MES cells (Fig. 2a). SK-N-HM also responded to BrdU by switching to an MES-like morphology and manifesting a significant reduction in telomerase activity. However, the change in telomere and DNA sensing protein levels in this cell line was slower and milder than those in $B E(2) N$ and $B E(2) C$ (Supp. Fig. 3d and 3e). Overall, our data indicate that the telomere-related differences observed between naturally derived ADRN and MES cell lines are recapitulated in pharmacologically generated ADRN and MES pairs.

\section{Stringent inhibition of telomerase activity triggers the reversible conversion of ADRN into MES cells}

The BrdU experiment showed that telomerase activity declined early and progressively during the conversion of ADRN cells into MES cells, suggesting that this activity may be involved in NB tumor cell differentiation, especially in MYCN-amplified tumor cells. To test this idea, we inhibited telomerase in the $\mathrm{BE}(2) \mathrm{N}$ cells by expressing a catalytically inactive, dominant negative allele of hTERT (Dn-hTERT) ${ }^{44}$. We generated amphotropic retroviruses carrying DnhTERT or wild-type hTERT, and infected BE(2)N cells with these viruses. Following drug selection, the cell populations were collected and passaged until day 100. Telomerase up-regulation and inhibition (in cell populations harboring hTERT and Dn-hTERT, respectively) were confirmed by the TRAP activity assays (Fig. 4a). In multiple, independently propagated Dn-hTERT-harboring cell populations, telomerase activity was greatly reduced, to $5 \%$ of the level found in parental $\mathrm{BE}(2) \mathrm{N}$ (day 55 samples in Fig. 4a and Supp. Fig. 4a). However, with further passaging, the activity became de-repressed (to $\sim 50 \%$ of the parental level or more). This de-repression may be due to a reduction in the level of Dn-hTERT mRNA, which was 8-fold lower on day 82 in comparison to day 55 as judged by allele-specific RT-PCR (Supp Fig. 4b). While the underlying reason for the reduction in Dn-hTERT mRNA is unclear, the resulting derepression of telomerase activity enabled us to examine the reversibility of the phenotypes induced by telomerase inhibition (see below). As expected, cells infected with the hTERT-bearing viruses manifested high levels of telomerase activity throughout passage (Fig. 4a and data not shown). 
We first characterized telomere lengths in the infected cells during passage. As anticipated, the increase in telomerase activity in hTERT-expressing cells caused telomere elongation, whereas the decrease in telomerase activity in Dn-hTERT-expressing cells caused telomere shortening (Fig. 4b). Surprisingly however, the de-repression of telomerase in late passages of Dn-hTERT-containing cells did not restore telomere length. Most interestingly, we found that telomerase inhibition can trigger the morphologic conversion of $B E(2) N$ from ADRN into MES-like cells (Supp. Fig. 4c). Similar to BrdU-induced conversion, the MES-like cells elicited by Dn-hTERT displayed the MES protein expression profile (Fig. 4c); the levels of telomere factors (TRF1 and TRF2) and MYCN were substantially reduced and that of STING was greatly increased at day 55 and 64. Remarkably, the time-dependent reversal in telomerase inhibition is matched by a similar reversion in cell morphology and protein expression profiles. In particular, low telomerase activity at day 55 and 64 correlated with MES cell morphology (enlarged and flattened with prominent vesicles) and protein profiles, whereas as high telomerase activity on day 77 and 91 was associated with the opposite features (Fig. 4c, Supp. Fig. 4c). Our observations strongly suggest that telomerase activity controls NB cell conversion in a reversible manner.

We also tested the effect of Dn-hTERT on $B E(2) C$, which resembles $B E(2) N$ phenotypically but is thought to possess more stem cell characteristics. However, telomerase inhibition in BE(2)C cells ( $70 \%$ inhibition) was not as efficient as that in $\mathrm{BE}(2) \mathrm{N}$, and telomerase activity was rapidly de-repressed to $>50 \%$ of the normal level found in parental cells (Supp Fig. 5). Notably, the Dn-hTERT-treated BE(2)C did not manifest morphologic conversion to MES-like cells, suggesting that stringent inhibition of telomerase is required for NB lineage switch. It is worth noting that in BrdUtreated $B E(2) N$, clear MES-like morphology did not emerge until day 20, when telomerase activity was less than $10 \%$ of untreated cells, again suggesting that stringent inhibition of telomerase is needed for full phenotypic conversion.

To characterize the genome-wide gene expression pattern during Dn-hTERT-induced lineage conversion, we compared the transcriptomes of Dn-hTERT-expressing cells at multiple time points by RNA-seq (Fig. 4d-4f). Similar to the transcriptomes of BrdU-treated $\mathrm{BE}(2) \mathrm{N}$, we found that telomerase inhibition induced progressive changes in gene expression patterns that correlated kinetically with changes in morphology and protein profiles. In particular, 
more genes were up- or down-regulated in Dn-hTERT-treated cells on day 55 (showing the greatest change in morphology) than on day 33 or day 82 (Fig. 4d). The heatmaps of individual genes further support progressive changes in the level of mRNAs from day 33 to day 55, as well as the reversal of expression on day 82 (Fig. 4 e). Also consistent with progressive change, comparisons of the RNA-seq data at different time points showed significant overlaps in the up-regulated and down-regulated gene lists between each pair of adjacent time points (i.e., between day 33 and day 55, as well as between day 55 and day 82, Fig. 4f). Moreover, the strong overlaps between the upand down-regulated gene sets on day 33 and day 82 indicate that morphologic reversion is due to the reversal of the transcription programs in these cells. Interestingly, comparison of the protein and RNA profiles suggests that some of the differences between cell lineages may be due to post-transcriptional regulation. For example, while the MYCN and STING mRNA levels changed in parallel with the respective proteins, the TRF1 and TRF2 mRNAs were unchanged throughout the Dn-hTERT-induced conversion process, suggesting that these telomere proteins are regulated at a post-transcriptional step (data not shown).

We next compared the up-regulated and down-regulated gene lists in the Dn-hTERT-induced MES-like cells (day 55) to the corresponding gene lists in BrdU-induced MES cells, and with previously defined MES and ADRN signature genes (Supp Fig. 6a) ${ }^{10}$. Strong overlaps were observed in each case, supporting the correspondence between BrdUand Dn-hTERT-induced MES cells, as well as the correspondence between these cells and previously defined MES. Gene ontology analysis of factors up-regulated by telomerase inhibition likewise support the involvement of MESrelated pathways such as cell adhesion and immune response (Supp. Fig. 6b). We also characterized the expression of a subset of ADRN and MES signature genes in our BrdU and Dn-hTERT experiments and found the ADRN genes to be consistently down-regulated by these treatments and the MES genes to be consistently up-regulated (Supp. Fig. 6c). Altogether, these analyses further support the designations of the different cell types in our study as ADRN and MES. 
To address the functional significance of the over-expression of DNA-sensing/innate immunity factors in MES cells, we infected two pairs of ADRN/MES cells with wild type Herpes Simplex Virus (KOS) or a mutant with altered cellular trafficking (vFH609) ${ }^{45}$. Indeed, both MES cell lines (LA1-5S and SH-EP1) exhibited robust response to KOS as evidenced by increased phosphorylation of TBK1 and STAT1, while the corresponding ADRN cells (LA1-55N and SHSY5Y) manifested little or no phosphorylation of these proteins (Fig. 5a and 5b). As expected from previous studies, vFH609 was less active than KOS in stimulating the phosphorylation cascade. Thus, the elevated levels of DNAsensing and innate immunity factors in MES evidently allow these cells to mount a stronger immunogenic response to DNA stimuli.

\section{Evidence for NB tumor cells with ADRN and MES expression profiles and relationship to prognosis}

To assess the relevance of the ADRN and MES cell lineages to NB tumors in patients, we queried the expression profiles of NB tumors in the Pediatric Neuroblastoma Target 2018 collection, specifically a set of tumors with RNAseq data ( $n=137)$ at cBioportal (https://www.cbioportal.org/) (see Table 1 for patient characteristics). Given the strong relationships between telomeres, cell lineage, and innate immunity in NB, the expression patterns (rank transformed) of 29 genes composed of key components of these pathways were used to identify tumors with predominantly ADRN or MES characteristics (Fig. 6a and 6b). Three clusters were uncovered through PAM clustering analysis, with clusters 1 and $3(n=35$ and $n=50)$ manifesting ADRN-like profiles (i.e., high telomere/low immunity/low MES marker expression) and cluster $2(n=52)$ manifesting an MES-like profile (i.e., low telomere/high immunity/high MES marker expression) (Table 1 and Fig. 6b). This finding supports the existence of NB tumors composed of predominantly one or the other cell types. We also analyzed the relative expression of specific telomere proteins against immunity and cell lineage markers in these samples and confirmed the expected positive and negative correlations (Supp. Fig. 7). For example, TRF2 expression is negatively correlated with STING and NOTCH3, but positively correlated with PHOX2B, precisely as predicted given the expression of these genes in MES and ADRN cell lines. We then explored the significance of cell lineages in disease progression by comparing the outcomes of patients belonging to different clusters (Fig. 6c). In univariate analysis, the cluster 1 and cluster 3 patients, despite both having predominantly ADRN-like profiles, manifested the best and worst overall survival (OS), respectively ( $p=$ 0.013). The MES-like cluster 2 patients had an intermediate outcome, although the difference between cluster 2 and 
$3(p=0.056)$ did not meet the threshold for statistical significance. Notably, after adjusting for age and stage of disease (two previously established prognostic variables), the survival differences between the different clusters were no longer statistically significant (data not shown). Together, these results suggest that NB cell lineage may be correlated with stage (regressing/differentiating local regional versus metastatic/relapsing) and age at diagnosis while influencing treatment outcome.

To identify differences between cluster 1 and 3 tumors that are associated with the disparity in outcome, we queried for genes that showed significant differences in RNA levels across the two clusters ( $p<0.01$, Wilcoxon rank sum test). Eight genes in our list of 29 were found to fit this criterion, and seven of these were related to telomere regulation (Supp. Table 1). Among the hits was TERT, which had previously been established as a strong prognostic indicator ${ }^{19}$. We further explored the significance of TERT expression by examining its predictive values in both univariate and multivariable analysis (adjusting for age and stage using Cox proportional hazards model), and observed better OS for patients with low levels of TERT (below median expression) in both analyses $(p<0.001$ and $p=0.021)$ (Fig. 6d). Similar results were obtained for event-free survival (EFS) (Fig. 6d), except that the EFS difference in multi-variable analysis was not statistically significant. Overall, these comparisons suggest that differences in TMM may be a key contributor to the differential outcomes of cluster 1 and 3 patients.

We also applied our gene signature analysis to a second group of patients (also in the Pediatric Neuroblastoma Target 2018 collection), whose tumors have been subjected to microarray profiling $(n=247)$. Using the same gene list and clustering method as described for the RNA-seq dataset, we identified four clusters of tumors for the microarray dataset: clusters 1, 2 and 4 are ADRN-like, and cluster 3 is MES-like (Supp. Fig. 8a and 8b). Again, two ADRN-like clusters, clusters 1 and 4, manifested the most benign and the poorest outcomes, respectively, while the MES-like cluster was intermediate in prognosis (Supp. Fig. 8c). Similar to results from the RNA-seq dataset, high TERT expression in the microarray dataset is associated with better EFS and OS, while low TERT expression is associated with poorer EFS and OS. Therefore, both TMM and tumor cell lineage may contribute to NB malignancy and could help refine prognosis. 


\section{Discussion}

The key discovery of the current study is the strong mechanistic connection between telomere regulation and tumor cell differentiation. The potential basis for this connection and its implications for the natural history of NB are discussed below.

\section{$\underline{\text { NB tumor cell lineage is reversibly regulated by telomerase activity independent of telomere lengths }}$}

One important outcome of this study is the discovery of a tight mechanistic relationship between telomerase activity and cell lineage in MYCN-amplified NB tumors. This relationship holds for both naturally-derived and experimentallygenerated ADRN/MES cell line pairs, with high telomerase indicative of the ADRN, and low telomerase indicative of the MES differentiation states, respectively. The Dn-hTERT experiment in particular, supports a causal role of telomerase activity in regulating NB differentiation. Interestingly, this does not seem to apply to non-MYCNamplified tumors, as evidenced by similar telomerase levels in the SH-SY5Y and SH-EP1 cells. The special importance of telomerase in MYCN-amplified tumors may be due to reciprocal effects of these proteins on each other's expression. In MYCN-amplified NB, MYCN is known to positively regulate TERT transcription ${ }^{14}$, and in this study we demonstrated a reciprocal, positive effect of telomerase activity on MYCN expression. Since MYCN has previously been implicated NB differentiation ${ }^{6}$, the positive feedback loop between MYCN and telomerase in this subset of high-risk tumors may explain the dependence of NB differentiation on telomerase. Conversely, we speculate that in non-MYCN-amplified tumors, MYCN and telomerase may be excluded from the gene expression network that regulates ADRN/MES switch. These considerations suggest that the specific genetic alterations that trigger NB tumorigenesis have an impact on differentiation program in the tumor.

Our discovery of the positive feedback relationship between MYCN and TERT reinforces the concept of "feed forward loop" in driving the reprogramming of NB tumor cell lineages ${ }^{9}$. Notably, inducible expression of multiple transcription factors, including NOTCH paralogs and PRRX1, have been shown to drive the conversion of ADRN into MES cells, arguing against the idea of a single master regulator ${ }^{9,10}$. Instead, factors activated during the conversion process may be mutually reinforcing, either through positive feedback of transcription (as we have demonstrated 
for MYCN and telomerase) or through alternative mechanisms such as mutual protein stabilization (e.g., see ${ }^{46}$ ). As pointed out earlier, this type of feed forward network has the advantage of enabling rapid transitions between two meta-stable states of differentiation ${ }^{9}$.

While the specific signals and pathways by which telomerase activity control MYCN expression and NB differentiation remain to be determined, the length of telomeres is evidently not an important factor. For example, in BrdU-treated $\mathrm{BE}(2) \mathrm{N}$ and $\mathrm{BE}(2) \mathrm{C}$ cells, the gradual conversion to MES cells paralleled the progressive loss in telomerase activity, yet no alterations in telomere lengths were observed. Likewise, the reversion to ADRN cells in late passages of DnhTERT-treated cells is accompanied by de-repression of telomerase activity but not any significant change in telomere lengths. Besides establishing the critical role of telomerase in differentiation, these results underscore the potential contributions of additional factors to telomere length homeostasis. Notably, both TRF1 and TRF2 are important regulators of telomere lengths, and both were down-regulated during BrdU and Dn-hTERT-induced cell lineage conversion, thereby complicating the relationship between telomerase activity and telomere lengths. TRF1 is part of the "protein-counting", negative feedback loop that regulates telomerase access to telomeres ${ }^{47,48}$. As such, low TRF1 level in MES cells may enable greater access of telomerase, thereby compensating for the reduction in telomerase level. TRF2, on the other hand, has been shown to regulate the extent of telomere loss; high TRF2 level is associated with faster and occasionally drastic telomere shortening ${ }^{49,50}$. Therefore, the low TRF2 level in MES could compensate for low telomerase activity by mitigating telomere loss. It will be interesting in the future to investigate whether these alterations in telomere protein levels are part of the normal mechanism that neural lineage cells utilize to maintain telomere lengths during neural development, when telomerase activity is known to undergo drastic changes ${ }^{35,51}$.

Consistent with our results, the inhibition of telomerase activity by Dn-hTERT in another MYCN-amplified cell line IGR-N-91 was reported to convert cells from a neuron-like morphology to one that resembles MES cells ${ }^{52}$. However, the repression of telomerase in this earlier study was stable, and no phenotypic reversion was observed. Because detailed transcriptional profiling was not available, it was unclear how similar the Dn-hTERT-induced cells were to the bone fide MES cell lineage. Nevertheless, when consider together with the current report, the data suggest that 
the tight causal relationship between telomerase and cell lineage may be applicable to MYCN-amplified NB tumors in general.

\section{The roles of telomere proteins in NB tumor cell reprogramming}

Telomerase is unlikely to be the only telomere factor involved in NB tumor cell differentiation, given the differences between ADRN and MES cells in the levels of many other telomere proteins. These include key components of the shelterin complex (TRF1, TRF2, TPP1, and POT1), as well as multiple factors involved in telomere maintenance (RTEL1 and ATRX). Indeed, previous studies have already implicated the double-strand telomere binding protein TRF2 in neural development, providing a plausible mechanism for how it could affect NB tumor cell differentiation. More specifically, TRF2 was shown to promote neural progenitor cell development and differentiation into mature neuron by interacting with REST (RE-1 silencing transcription factor) and antagonizing its neural repressive function $31,32,33$. In support of this non-telomeric mechanism, we detected significant cytoplasmic localization of TRF2 in ADRN cells. Interestingly, inspection of RNA-seq data revealed up-regulation of REST mRNA in both BrdU- and Dn-hTERTinduced MES cells, supporting the potential involvement of the TRF2-REST pathway in NB differentiation. Therefore, like telomerase, TRF2 and other telomere proteins may also be part of the feed forward network that promote NB lineage conversion. Yet another differentially expressed telomere protein that can significantly affect disease biology is ATRX, a chromatin remodeling factor frequently inactivated in ALT-positive NB. Loss of ATRX is believed to trigger progressive telomere replication dysfunction, culminating ultimately in the activation of ALT during immortalization

53. We found that ATRX is dramatically down-regulated in MES cells, suggesting that these cells may be prone to telomere dysfunction and to the activation of ALT. Beyond NB, ALT is known to occur at higher frequencies in cancers of mesenchymal origin ${ }^{23}$. Whether this is linked to down-regulation of ATRX is an interesting question for future investigation.

Previous analyses of ADRN and MES cells have not revealed strong differences in the levels of telomere-related factors. Our preliminary data suggests that some of the differences in protein levels may be due to post transcriptional regulation, and therefore overlooked in transcriptomic analysis. For example, RNA-seq data indicate that the levels of TRF1 and ATRX mRNA in $\mathrm{BE}(2) \mathrm{N}$ was unchanged following BrdU treatment, in clear contrast to the 
drastic reduction in protein levels. Previous studies of neuro-progenitor cells also suggest significant posttranscriptional regulation of telomere-related genes during neural differentiation ${ }^{33}$. In addition, the discordance between ATRX mRNA and protein levels is supported by recent profiling of ALT-positive NB tumors ${ }^{54}$. More detailed proteomic analysis of different cell lineages may be required to gain a full understanding of the phenotypic differences between these lineages.

\section{Implications for NB prognosis and therapy}

Both telomere maintenance and tumor cell lineage have been implicated in the prognosis of NB, each with plausible underlying mechanisms. Telomere DNA is subject to continuous erosion and drastic truncation owing to incomplete replication and telomere trimming. Without compensatory mechanisms, the loss of telomere DNA should eventually trigger a DNA damage response that compromises tumor cell proliferation. Not surprisingly, in two large-scale studies of NB tumor samples, the presence of either high telomerase activity or ALT activity is strongly associated with poor prognosis ${ }^{18,19}$. The role of NB tumor cell differentiation in disease progression is also plausible but mechanistically complex. Initial studies suggest that MES cells may be less tumorigenic in mice ${ }^{7}$, whereas a more recent study reported comparable malignancy of matched ADRN/MES cell lines generated by controlled expression of NOTCH3-IC ${ }^{9}$. Importantly, these studies did not take into account the potential effects of host immune response, and growing evidence suggests that ADRN and MES cells differ dramatically in their ability to induce immunity and inflammation. Wolpaw et al. report elevated TLR signaling in natural and induced MES cells; upon stimulation, these cells secrete high levels of pro-inflammatory cytokines, leading to increased tumor cell killing by T-cells in vitro ${ }^{37}$. Similarly, Sengupta et al. demonstrated enrichment of innate and adaptive immune gene signature in MES cells, and showed that these cells not only engaged cytotoxic and natural killer cells, but also induced immune cell infiltration in immunocompetent mice ${ }^{36}$. Echoing these results, we show in our analysis strong up-regulation of several key components of the DNA sensing/innate immunity pathway in naturally derived as well as BrdU- and Dn-hTERTinduced MES cells. In addition, the high levels of cGAS, STING and IRF3 in MES cells evidently enable these cells to generate a stronger response to viral DNA challenge. Together, these studies highlight a strong linkage between telomere-remodeling, cell lineage re-programming, and alterations in immunogenicity, which have strong implications for developing new biomarkers and therapies for this aggressive pediatric cancer. Indeed, by using a 
gene signature that incorporates differentially expressed genes related to telomere regulation, cell lineage, and immunogenicity, we were able to identify three clusters of NB tumors that manifest either a predominantly MESlike or ADRN-like phenotype. The different survival statistics for patients in different clusters illustrates the potential prognostic value of genes associated with these pathways. Most importantly, the strong interconnections between telomeres, cell lineage and immunity suggest that there may be many targets in the differentiation network of NB that can be manipulated to improve therapeutic response. In this regard, we suggest that inhibiting telomerase in MYCN-amplified tumors may be an especially potent strategy - by simultaneously eliminating telomere maintenance and enhancing immunogenicity through tumor cell lineage reprogramming.

\section{Methods}

\section{Cell culture}

Neuroblastoma cell lines SK-N-BE(2)N (abbreviated as BE(2)N), SK-N-BE(2)C (abbreviated as BE(2)C), and SK-N-HM were originally established at MSKCC, while LA1-55N, LA1-5S, LA1-66N, LA1-6S, SH-SY5Y, and SH-EP1 cell lines were obtained from Robert Ross. These cell lines were cultured in RPMI-1640 medium (CORING) supplemented with $10 \%$ fetal bovine serum (FBS) (GEMINI) and 1\% penicillin/streptomycin (Pen/Strep) (Gibco). The human cervical cancer cell line HeLa wase also obtained from ATCC, and was cultured in Dulbecco's modified Eagle's medium (DMEM) (CORING) supplemented with $10 \%$ FBS and $1 \%$ Pen/Strep. Phoenix cell line for retrovirus packaging was kindly provided by Dr. Xin-Yun Huang at Weill Cornell Medicine, and was cultured in DMEM supplemented with $10 \%$ FBS, $1 \%$ Pen/Strep, and $1 \%$ glutamine.

\section{Production of retrovirus stocks and infections of NB cell lines}

pBABE-puro-hTERT and pBABE-puro-Dn-hTERT plasmid (dominant negative hTERT) was provided by Dr. Duncan Baird (Cardiff University, U.K.) ${ }^{44}$. Amphotropic retroviruses were generated in Phoenix packaging cells by $\mathrm{Ca}_{2} \mathrm{PO}_{4}$ transfection of the retroviral vectors ( $20 \mu \mathrm{g}$ each) using protocols described by Dr. Garry Nolan (Stanford University) and Dr. Titia de Lange (Rockefeller University). Transfection efficiency was monitored based on GFP expression. At $48 \mathrm{hr}$ post-transfection, the viral supernatants were filtered through a $0.45 \mu \mathrm{m}$ filter and used to infect $\mathrm{BE}(2) \mathrm{N}$ and 
$\mathrm{BE}(2) \mathrm{C}$ cells with the addition of $4 \mu \mathrm{g} / \mathrm{ml}$ polybrene. Cells were infected twice for $6 \sim 8 \mathrm{hr}$, and were selected with puromycin $(0.9 \mu \mathrm{g} / \mathrm{ml}$ for $\mathrm{BE}(2) \mathrm{N}$ and $0.75 \mu \mathrm{g} / \mathrm{ml}$ for $\mathrm{BE}(2) \mathrm{C}))$ at $48 \mathrm{hr}$ post-infection.

\section{Western}

PVDF membrane filters with transferred proteins were blocked with TTBS containing 5\% non-fat milk, and incubated with the following primary antibodies: mouse anti-TRF1 (SCBT, sc-56807), mouse anti-TRF2 (Novusbio, NB10056506), mouse anti-RAP1 (SCBT, sc-53434), mouse anti-TIN2 (Novusbio, NB600-1522), rabbit anti-TPP1 (Bethyl, A303-069A-T), rabbit anti-POT1 (Novusbio, NB500-176), mouse anti-GAPDH (ABclonal, AC002), mouse anti-TERT (SCBT, sc-393013), mouse anti-STN1/OBFC1 (SCBT, sc-374178), mouse anti-PRIM1 (SCBT, sc-390265), mouse antiPOLA2 (SCBT, sc-398255), mouse anti-RPA70 (SCBT, sc-48425), rabbit anti-TZAP (Proteintech, 24665-1-AP), mouse anti-ATRX (SCBT, sc-55584), mouse anti-RTEL1 (SCBT, sc-515427), mouse anti-RIF1 (SCBT, sc-515573), mouse antiMYCN (SCBT, sc-53993), mouse anti-IRF3 (SCBT, sc-33641), rabbit anti-STING (Cell Signaling, \#13647), mouse anticGAS (SCBT, sc-515777), mouse anti-SLUG (SCBT, sc-166476), mouse anti-GATA3 (SCBT, sc-269), rabbit antiphospho-STAT1 (Cell Signaling, \#9167), rabbit anti-phospho-TBK1 (Cell Signaling, \#5384). Secondary antibodies were either HRP-linked anti-rabbit IgG (Cell Signaling, \#7074) or HRP-linked Anti-Mouse IgG (Cell Signaling, \#7076), and were applied to the filters in TTBS containing 5\% non-fat milk. After washing, the blots were visualized using chemiluminescence reagents (Tanon ${ }^{\mathrm{TM}}$ High-sig ECL Western Blotting Substrate, ABclonal).

\section{$\underline{I F-F I S H}$}

Immunofluorescence (IF) combined with fluorescence in situ hybridization (FISH) was performed as previously described with minor modifications ${ }^{55}$. Briefly, LA1-55N, LA1-5S, and HeLa cells were seeded at $7 \times 10^{5}, 4 \times 10^{5}$, or 3 x $10^{5}$ cells per well, respectively, in 6-well plates containing coverslips with Poly-D-Lysine (PDL) coating (Neuvitro Corp.), and cells were cultured for 24 hours. Cells were fixed with $4 \%$ paraformaldehyde in PBS for 10 min, permeabilized with ice-cold $0.5 \%$ Triton $\mathrm{X}-100$ on ice for $5 \mathrm{~min}$, and stained for protein antigen overnight at $4^{\circ} \mathrm{C}$ in a humidified chamber. The following primary antibody were used: mouse- $\alpha$-TRF1 (TRF-78) (ab10579, Abcam), mouse$\alpha$-TRF2 (NB100-56506, Novus Biologicals). Goat- $\alpha-m o u s e-A l e x a-488$ (A28175, Invitrogen) was used as the secondary antibody. Cells were fixed again with 4\% paraformaldehyde in PBS for 6 min, and then subjected to FISH using a 
peptide nucleic acid (PNA) probe specific to the telomeric sequence (CCCTAA) 3 (TelC-Cy3, PNA BIO). Cells were dehydrated successively in $70 \%, 90 \%$, and $100 \%$ ethanol and air dried, and nuclear DNA was denatured for 10 min at $85{ }^{\circ} \mathrm{C}$ in hybridization buffer containing $1.25 \mu \mathrm{g} / \mathrm{ml}\left(\mathrm{C}_{3} \mathrm{TA}_{2}\right)_{3}-\mathrm{Cy} 3$-labeled PNA telomeric probe, $70 \%$ formamide, $0.5 \%$ blocking reagent (Roche), $10 \mathrm{mM}$ Tris- $\mathrm{HCl}$ ( $\mathrm{pH} 7.4)$. After denaturation, incubation was continued overnight at $4{ }^{\circ} \mathrm{C}$ in a humidified chamber. Cells were washed twice, each for $15 \mathrm{~min}$ with $70 \%$ formamide/10 mM Tris- $\mathrm{HCl}(\mathrm{pH}$ 7.4), followed by a 5 min wash with PBS three times. After air-drying, the coverslips were placed on top of embedding medium (ProLong Gold antifade reagent with DAPI, Invitrogen) on a slide and sealed with nail polish. FLUOVIEW FV10i (OLYMPUS) microscope was used to capture images. For BrdU-treated BE(2)N, cells were cultured with $7.5 \mu \mathrm{M}$ of BrdU for up to 16 days. On the day before the IF-FISH procedure, cells were seeded at $7.5 \times 10^{5}$ cells per well in 6 well plates containing coverslips with PDL coating. The cells were allowed to grow on the coverslips for another 24 $\mathrm{hr}$, and then subjected to IF-FISH.

\section{Immune activation}

KOS and vH609 virus particles were prepared as described ${ }^{45}$. Neuroblastoma cells were cultured in a 12-well dish to a density of $\sim 5 \times 10^{5}$ cells per well, and then treated with KOS or vFH609 virus particles at a titer of $\sim 3 \times 10^{3}$ virus particles per cell. Cells were collected $4 \mathrm{~h}$ after infection, and extracts were subjected to Western analysis.

\section{$\underline{R N A-s e q}$}

RNA-sequencing was performed on the following human NB cell lines: LA1-66N, LA1-6S, BE(2)N (untreated), BE(2)N (Dn-hTERT treated - on day 33, day 55, and day 82), BE(2)N (DMSO-treated), and BE(2)N (BrdU treated - on day 5, day 11 , day 20 , and day 26). Total RNA was isolated with the RNeasy Mini kit (Qiagen), and the quality of the preparations assessed on an Agilent 2100 Bioanalyzer (Agilent Technologies). TruSeq stranded mRNA libraries were prepared and sequenced with paired-end 50 bps on Illumina NovaSeq 6000 by the WCM Genomics Core Facility. The raw sequencing reads in BCL format were processed through bcl2fastq 2.19 (Illumina) for FASTQ conversion and demultiplexing. RNA reads were aligned and mapped to theGRCh37 human reference genome by STAR (Version2.5.2) (https://github.com/alexdobin/STAR) ${ }^{56}$, and transcriptome reconstruction was performed by Cufflinks (Version 2.1.1) (http://cole-trapnell-lab.github.io/cufflinks/). The abundance of transcripts was measured with Cufflinks in 
Fragments Per Kilobase of exon model per Million mapped reads (FPKM) ${ }^{57,58}$. The fold changes were calculated by directly comparing the FPKM values of samples and controls.

Up- and down-regulated genes were clustered and displayed as heatmaps using ClustVis ${ }^{59}$. For identification of overlaps between gene sets, the lists were uploaded and analyzed at the Bioinformatics Evolutionary Genomics server (http://bioinformatics.psb.ugent.be/webtools/Venn/). Statistical significance values were calculated using the Nematode Bioinformatics server (http://nemates.org/MA/progs/overlap_stats.html). The data series have been uploaded to GEO (accession GSE171404).

\section{$\underline{R T-P C R}$}

Total RNA was isolated from Dn-hTERT expressing BE(2)N cells collected at days 33, 55, and 82 (D33, D55, D82), using the RNeasy Mini kit (Qiagen). Purified RNA was reverse transcribed to cDNA using Transcriptor Reverse Transcriptase (Roche), and the cDNA products subjected to PCR using $1 \mu$ cDNA, 2.5 U Choice-Taq Blue DNA polymerase (Denville) and PCR primers $(2.5 \mu \mathrm{M})$ in a total volume of $20 \mu \mathrm{l}$. Each cDNA obtained from Dn-TERT expressing cells were subjected to serial dilutions to facilitate quantitative comparison. Relative expressions of mRNAs (for both wild type and Dn-hTERT) were calculated by comparing the intensities and controlling for dilution factors using GraphPad Prism.

\section{Assays of telomeres and telomerase activity (TRF Southern, STELA, in-gel hybridization, TRAP)}

Telomere restriction fragment (TRF) Southern analysis was performed as described except that we used a probe comprised of 82 copies of the TTAGGG repeats ${ }^{60}$. For quantitative analysis of TRF length distribution and determination of average telomere lengths, we used the weighed average method as implemented by Kimura et al. ${ }^{61}$ and Mender and Shay ${ }^{62}$. STELA and in-gel hybridization were carried out exactly as previously described ${ }^{27}$. The telomere repeat amplification protocol (TRAP) was performed according to a published protocol ${ }^{63}$ except that $\mathrm{P}^{32}$ labeled TS primers were used and the signals were quantified on a Phosphor scanner (FLA7000, GE Healthcare). To determine relative telomerase levels between samples, we first calculated the ratio of TRAP signals to the control PCR product (IC) for each assay. A standard curve that relates extract amounts to the TRAP/IC ratios was then 
constructed using the dilution series that yielded the highest signals in a given gel. (Typically the curve is close to linear or hyperbolic.) A series of XY values were then generated from the curve using PRISM, which allowed us to determine the equivalent amount of extract needed to yield the specific TRAP/IC signals produced by other samples in the gel. Multiplying this value by the dilution factor provides the relative telomerase activity level in a given extract.

\section{$\underline{\text { NB tumor database analysis }}$}

The Pediatric Neuroblastoma (Target, 2018) tumor collection at cBioportal were profiled with respect to the mRNA levels of 29 genes implicated in telomere, cell lineage, and immune regulation and that showed strong differential expression in ADRN and MES cells (Fig. 6b). Tumors with RNA-seq $(n=137)$ and microarray $(n=247)$ expression data were analyzed separately. Gene expression levels were rank transformed and clustered using Partition around medoids. The optimal numbers of clusters were determined based on the average silhouette width and strength of association with patient overall survival (OS) and were found to be 3 and 4 for the RNA-seq and microarray datasets, respectively. The relationships between the expression clusters and patients' overall and event free survival (EFS) were evaluated using log-rank test. To identify genes whose expression showed consistent differences between cluster 1 and 3 patients, we applied the non-parametric Wilcoxon rank sum test to each of the 29 genes in the signature and used $\mathrm{P}<0.01$ for both tests as the cutoff (Supp. Table 1). To assess the association between TERT levels and survival, TERT RNA levels were converted to a categorical variable (high TERT and low TERT) dichotomized at the median. Overall survival and event free survival were then examined in (i) univariate analysis using log-rank test and (ii) multivariable analysis adjusting for age and stage using Cox proportional hazards model. These analyses were carried out using R 4.0.4 (https://r-project.org).

\section{$\underline{\text { Statistics }}$}

For experiments related to NB cell lines, GraphPad Prism was used to graph data as mean \pm SD and to calculate P values using Student's t test (two-tailed). The detailed statistical methods used for the analysis of the Pediatric Neuroblastoma (Target, 2018) tumor collection were described in the relevant section above.

\section{Data and code availability}


The datasets generated and/or analyzed during the current study are included within the paper and Supplementary Information or available from the corresponding author upon reasonable request. The RNA-seq data associated with Fig. 3, Fig. 4 and Supp. Fig. 6 have been uploaded to GEO (accession GSE171404). All the software used in this study is publicly available.

\section{Acknowledgement}

We thank members of our laboratories for comments. This work was supported by a Seed Grant for Collaborative Multi-Investigator Projects between Cornell University Ithaca, Weill Cornell Medicine, and Cornell Tech. N.F.L is a recipient of the William Randolph Hearst Endowed Faculty Fellowship in Microbiology. X.K.Z. is supported in part by 1UL1 TR002384-01 to Weill Cornell Clinical and Translational Science Center. 


\section{References}

1. Cheung NKV, Dyer MA. Neuroblastoma: developmental biology, cancer genomics and immunotherapy. Nat Rev Cancer 13, 397-411 (2013).

2. Louis CU, Shohet JM. Neuroblastoma: molecular pathogenesis and therapy. Annu Rev Med 66, 49-63 (2015).

3. Dong R, et al. Single-Cell Characterization of Malignant Phenotypes and Developmental Trajectories of Adrenal Neuroblastoma. Cancer Cell 38, 716-733 e716 (2020).

4. Kildisiute $\mathrm{G}$, et al. Tumor to normal single-cell mRNA comparisons reveal a pan-neuroblastoma cancer cell. Sci Adv 7, (2021).

5. Ciccarone V, Spengler BA, Meyers MB, Biedler JL, Ross RA. Phenotypic diversification in human neuroblastoma cells: expression of distinct neural crest lineages. Cancer Res 49, 219-225 (1989).

6. Ross RA, Spengler BA, Domenech C, Porubcin M, Rettig WJ, Biedler JL. Human neuroblastoma I-type cells are malignant neural crest stem cells. Cell Growth Differ 6, 449-456 (1995).

7. Walton JD, et al. Characteristics of stem cells from human neuroblastoma cell lines and in tumors. Neoplasia 6, 838-845 (2004).

8. Acosta $\mathrm{S}$, et al. Comprehensive characterization of neuroblastoma cell line subtypes reveals bilineage potential similar to neural crest stem cells. BMC Dev Biol 9, 12 (2009).

9. van Groningen T, et al. A NOTCH feed-forward loop drives reprogramming from adrenergic to mesenchymal state in neuroblastoma. Nat Commun 10, 1530 (2019).

10. van Groningen $\mathrm{T}$, et al. Neuroblastoma is composed of two super-enhancer-associated differentiation states. Nat Genet 49, 1261-1266 (2017).

11. Boeva $\mathrm{V}$, et al. Heterogeneity of neuroblastoma cell identity defined by transcriptional circuitries. Nat Genet 49, 1408-1413 (2017). 
12. Wezel EMv, et al. Mesenchymal Neuroblastoma Cells Are Undetected by Current mRNA Marker Panels: The Development of a Specific Neuroblastoma Mesenchymal Minimal Residual Disease Panel. JCO Precision Oncology, 1-11 (2019).

13. Yogev O, et al. In Vivo Modeling of Chemoresistant Neuroblastoma Provides New Insights into Chemorefractory Disease and Metastasis. Cancer Res 79, 5382-5393 (2019).

14. Peifer $\mathrm{M}$, et al. Telomerase activation by genomic rearrangements in high-risk neuroblastoma. Nature 526, 700-704 (2015).

15. Hertwig F, Peifer M, Fischer M. Telomere maintenance is pivotal for high-risk neuroblastoma. Cell Cycle 15, 311-312 (2016).

16. de Lange T. Shelterin-Mediated Telomere Protection. Annu Rev Genet 52, 223-247 (2018).

17. Shay JW. Role of Telomeres and Telomerase in Aging and Cancer. Cancer Discov 6, 584-593 (2016).

18. Ackermann S, et al. A mechanistic classification of clinical phenotypes in neuroblastoma. Science 362, 1165-1170 (2018).

19. Koneru B, et al. Telomere Maintenance Mechanisms Define Clinical Outcome in High-Risk Neuroblastoma. Cancer Res 80, 2663-2675 (2020).

20. Wu RA, Upton HE, Vogan JM, Collins K. Telomerase Mechanism of Telomere Synthesis. Annu Rev Biochem 86, 439-460 (2017).

21. Autexier C, Lue NF. The structure and function of telomerase reverse transcriptase. Annu Rev Biochem 75, 493-517 (2006).

22. Pickett HA, Reddel RR. Molecular mechanisms of activity and derepression of alternative lengthening of telomeres. Nat Struct Mol Biol 22, 875-880 (2015).

23. Dilley RL, Greenberg RA. ALTernative Telomere Maintenance and Cancer. Trends Cancer 1, 145-156 (2015). 
24. Valentijn $\amalg$, et al. TERT rearrangements are frequent in neuroblastoma and identify aggressive tumors. Nat Genet 47, 1411-1414 (2015).

25. Pickett HA, Henson JD, Au AY, Neumann AA, Reddel RR. Normal mammalian cells negatively regulate telomere length by telomere trimming. Hum Mol Genet 20, 4684-4692 (2011).

26. Rivera T, Haggblom C, Cosconati S, Karlseder J. A balance between elongation and trimming regulates telomere stability in stem cells. Nat Struct Mol Biol 24, 30-39 (2017).

27. Yu EY, et al. Telomere Trimming and DNA Damage as Signatures of High Risk Neuroblastoma. Neoplasia 21, 689-701 (2019).

28. Arnoult N, Karlseder J. Complex interactions between the DNA-damage response and mammalian telomeres. Nat Struct Mol Biol 22, 859-866 (2015).

29. George SL, et al. Novel therapeutic strategies targeting telomere maintenance mechanisms in high-risk neuroblastoma. J Exp Clin Cancer Res 39, 78 (2020).

30. Huang M, et al. Epigenetic Targeting of TERT-Associated Gene Expression Signature in Human Neuroblastoma with TERT Overexpression. Cancer Res 80, 1024-1035 (2020).

31. Zhang $\mathrm{P}$, et al. Nontelomeric splice variant of telomere repeat-binding factor 2 maintains neuronal traits by sequestering repressor element 1-silencing transcription factor. Proceedings of the National Academy of Sciences of the United States of America 108, 16434-16439 (2011).

32. Zhang $\mathrm{P}$, et al. Nontelomeric TRF2-REST interaction modulates neuronal gene silencing and fate of tumor and stem cells. Curr Biol 18, 1489-1494 (2008).

33. Ovando-Roche P, Yu JS, Testori S, Ho C, Cui W. TRF2-mediated stabilization of hREST4 is critical for the differentiation and maintenance of neural progenitors. Stem Cells 32, 2111-2122 (2014).

34. Fu W, Killen M, Culmsee C, Dhar S, Pandita TK, Mattson MP. The catalytic subunit of telomerase is expressed in developing brain neurons and serves a cell survival-promoting function. $J$ Mol Neurosci 14, 315 (2000). 
35. Mattson MP, Klapper W. Emerging roles for telomerase in neuronal development and apoptosis. $J$ Neurosci Res 63, 1-9 (2001).

36. Sengupta $\mathrm{S}$, et al. Cell lineage as a predictor of immune response in neuroblastoma. bioRxiv, 2021.2001.2029.428154 (2021).

37. Wolpaw AJ, et al. Epigenetic state determines inflammatory sensing in neuroblastoma. bioRxiv, 2021.2001.2027.428523 (2021).

38. Lue NF, Chan J, Wright WE, Hurwitz J. The CDC13-STN1-TEN1 complex stimulates Pol alpha activity by promoting RNA priming and primase-to-polymerase switch. Nat Commun 5, 5762 (2014).

39. Derboven E, Ekker H, Kusenda B, Bulankova P, Riha K. Role of STN1 and DNA polymerase alpha in telomere stability and genome-wide replication in Arabidopsis. PLoS Genet 10, e1004682 (2014).

40. Abdisalaam $S$, et al. Dysfunctional telomeres trigger cellular senescence mediated by cyclic GMP-AMP synthase. J Biol Chem 295, 11144-11160 (2020).

41. Chen YA, Shen YL, Hsia HY, Tiang YP, Sung TL, Chen LY. Extrachromosomal telomere repeat DNA is linked to ALT development via cGAS-STING DNA sensing pathway. Nat Struct Mol Biol 24, 1124-1131 (2017).

42. Oganesian L, Karlseder J. 5' C-rich telomeric overhangs are an outcome of rapid telomere truncation events. DNA Repair (Amst) 12, 238-245 (2013).

43. Lazzerini-Denchi E, Sfeir A. Stop pulling my strings - what telomeres taught us about the DNA damage response. Nat Rev Mol Cell Biol 17, 364-378 (2016).

44. Hahn WC, et al. Inhibition of telomerase limits the growth of human cancer cells. Nat Med 5, 1164-1170 (1999).

45. Huffman JB, et al. The C Terminus of the Herpes Simplex Virus UL25 Protein Is Required for Release of Viral Genomes from Capsids Bound to Nuclear Pores. J Virol 91, (2017).

46. Koh CM, et al. Telomerase regulates MYC-driven oncogenesis independent of its reverse transcriptase activity. J Clin Invest 125, 2109-2122 (2015). 
47. van Steensel B, de Lange T. Control of telomere length by the human telomeric protein TRF1. Nature 385, 740-743 (1997).

48. Loayza D, De Lange T. POT1 as a terminal transducer of TRF1 telomere length control. Nature 423, 10131018 (2003).

49. Karlseder J, Smogorzewska A, de Lange T. Senescence induced by altered telomere state, not telomere loss. Science 295, 2446-2449 (2002).

50. Nera B, Huang HS, Lai T, Xu L. Elevated levels of TRF2 induce telomeric ultrafine anaphase bridges and rapid telomere deletions. Nat Commun 6, 10132 (2015).

51. Klapper W, Shin T, Mattson MP. Differential regulation of telomerase activity and TERT expression during brain development in mice. J Neurosci Res 64, 252-260 (2001).

52. Samy $M$, et al. Loss of the malignant phenotype of human neuroblastoma cells by a catalytically inactive dominant-negative hTERT mutant. Mol Cancer Ther 11, 2384-2393 (2012).

53. Li F, et al. ATRX loss induces telomere dysfunction and necessitates induction of alternative lengthening of telomeres during human cell immortalization. EMBO J 38, e96659 (2019).

54. Hartlieb SA, et al. Alternative lengthening of telomeres in childhood neuroblastoma from genome to proteome. Nat Commun 12, 1269 (2021).

55. Herbig U, Jobling WA, Chen BP, Chen DJ, Sedivy JM. Telomere shortening triggers senescence of human cells through a pathway involving ATM, p53, and p21(CIP1), but not p16(INK4a). Mol Cell 14, 501-513 (2004).

56. Dobin A, et al. STAR: ultrafast universal RNA-seq aligner. Bioinformatics 29, 15-21 (2013).

57. Trapnell C, Hendrickson DG, Sauvageau M, Goff L, Rinn JL, Pachter L. Differential analysis of gene regulation at transcript resolution with RNA-seq. Nat Biotechnol 31, 46-53 (2013).

58. Trapnell C, et al. Transcript assembly and quantification by RNA-Seq reveals unannotated transcripts and isoform switching during cell differentiation. Nat Biotechnol 28, 511-515 (2010). 
59. Metsalu T, Vilo J. ClustVis: a web tool for visualizing clustering of multivariate data using Principal Component Analysis and heatmap. Nucleic Acids Res 43, W566-570 (2015).

60. de Lange T, et al. Structure and variability of human chromosome ends. Mol Cell Biol 10, 518-527 (1990).

61. Kimura $M$, et al. Measurement of telomere length by the Southern blot analysis of terminal restriction fragment lengths. Nature Protocols 5, 1596-1607 (2010).

62. Mender I, Shay JW. Telomere Restriction Fragment (TRF) Analysis. Bio Protoc 5, (2015).

63. Mender I, Shay JW. Telomerase Repeated Amplification Protocol (TRAP). Bio Protoc 5, (2015). 
bioRxiv preprint doi: https://doi.org/10.1101/2021.05.09.442765; this version posted May 10, 2021. The copyright holder for this preprint (which was not certified by peer review) is the author/funder. All rights reserved. No reuse allowed without permission.

Table 1. Patient characteristics in the three gene expression clusters

\begin{tabular}{|c|c|c|c|c|c|}
\hline & All $(n=137)$ & $1(n=35)$ & $2(n=52)$ & $3(n=50)$ & $\begin{array}{l}\mathrm{p}- \\
\text { values* }\end{array}$ \\
\hline \multicolumn{6}{|l|}{ AGE } \\
\hline Mean+/-sd & $3.99+/-2.89$ & $2.91+/-2.29$ & $4.1+/-3.42$ & $4.62+/-2.46$ & \\
\hline Median (IQR) & $3(2,5)$ & $2(1,4)$ & $3(2,5)$ & $4(3,6)$ & 0.001 \\
\hline \multicolumn{6}{|l|}{ SEX, n (\%) } \\
\hline Female & $57(41.61 \%)$ & $8(22.86 \%)$ & $25(48.08 \%)$ & $24(48 \%)$ & \\
\hline Male & $80(58.39 \%)$ & $27(77.14 \%)$ & $27(51.92 \%)$ & $26(52 \%)$ & 0.031 \\
\hline \multicolumn{6}{|l|}{ RACE, $n$ (\%) } \\
\hline Asian & $1(0.73 \%)$ & $0(0 \%)$ & $0(0 \%)$ & $1(2 \%)$ & \\
\hline Black or African American & $24(17.52 \%)$ & $8(22.86 \%)$ & $7(13.46 \%)$ & $9(18 \%)$ & \\
\hline $\begin{array}{l}\text { Native Hawaiian or other Pacific } \\
\text { Islander }\end{array}$ & $2(1.46 \%)$ & $0(0 \%)$ & $0(0 \%)$ & $2(4 \%)$ & \\
\hline Not Reported & $3(2.19 \%)$ & $1(2.86 \%)$ & $1(1.92 \%)$ & $1(2 \%)$ & \\
\hline Unknown & $10(7.3 \%)$ & $4(11.43 \%)$ & $2(3.85 \%)$ & $4(8 \%)$ & \\
\hline White & $97(70.8 \%)$ & $22(62.86 \%)$ & $42(80.77 \%)$ & $33(66 \%)$ & 0.528 \\
\hline \multicolumn{6}{|l|}{ ETHNICITY, n (\%) } \\
\hline Hispanic or Latino & $12(8.76 \%)$ & $4(11.43 \%)$ & $3(5.77 \%)$ & $5(10 \%)$ & \\
\hline Not Hispanic or Latino & $114(83.21 \%)$ & $27(77.14 \%)$ & $44(84.62 \%)$ & $43(86 \%)$ & \\
\hline Unknown & $11(8.03 \%)$ & $4(11.43 \%)$ & $5(9.62 \%)$ & $2(4 \%)$ & 0.554 \\
\hline \multicolumn{6}{|l|}{ INSS_STAGE, n (\%) } \\
\hline Stage $2 b$ & $1(0.73 \%)$ & $1(2.86 \%)$ & $0(0 \%)$ & $0(0 \%)$ & \\
\hline Stage 3 & $5(3.65 \%)$ & $3(8.57 \%)$ & $1(1.92 \%)$ & $1(2 \%)$ & \\
\hline Stage 4 & $111(81.02 \%)$ & $20(57.14 \%)$ & $43(82.69 \%)$ & $48(96 \%)$ & \\
\hline Stage $4 \mathrm{~s}$ & $20(14.6 \%)$ & $11(31.43 \%)$ & $8(15.38 \%)$ & $1(2 \%)$ & $<0.001$ \\
\hline \multicolumn{6}{|l|}{ TUMOR_SAMPLE_HISTOLOGY, n (\%) } \\
\hline Favorable & $25(18.25 \%)$ & $12(34.29 \%)$ & $10(19.23 \%)$ & $3(6 \%)$ & \\
\hline Unfavorable & $102(74.45 \%)$ & $21(60 \%)$ & $36(69.23 \%)$ & $45(90 \%)$ & \\
\hline Unknown & $10(7.3 \%)$ & $2(5.71 \%)$ & $6(11.54 \%)$ & $2(4 \%)$ & 0.005 \\
\hline \multicolumn{6}{|l|}{ DIAGNOSIS, n (\%) } \\
\hline Ganglioneuroblastoma, intermixed & $1(0.73 \%)$ & $0(0 \%)$ & $1(1.92 \%)$ & $0(0 \%)$ & \\
\hline Ganglioneuroblastoma, nodular & $22(16.06 \%)$ & $5(14.29 \%)$ & $10(19.23 \%)$ & $7(14 \%)$ & \\
\hline Neuroblastoma & $109(79.56 \%)$ & $27(77.14 \%)$ & $40(76.92 \%)$ & $42(84 \%)$ & \\
\hline Unknown & $5(3.65 \%)$ & $3(8.57 \%)$ & $1(1.92 \%)$ & $1(2 \%)$ & 0.567 \\
\hline \multicolumn{6}{|l|}{ RISK_GROUP, n (\%) } \\
\hline High Risk & $113(82.48 \%)$ & $20(57.14 \%)$ & $44(84.62 \%)$ & 49 (98\%) & \\
\hline
\end{tabular}


bioRxiv preprint doi: https://doi.org/10.1101/2021.05.09.442765; this version posted May 10, 2021. The copyright holder for this preprint (which was not certified by peer review) is the author/funder. All rights reserved. No reuse allowed without permission.

\begin{tabular}{|c|c|c|c|c|c|}
\hline Intermediate Risk & $10(7.3 \%)$ & $8(22.86 \%)$ & $1(1.92 \%)$ & $1(2 \%)$ & \\
\hline Low Risk & $14(10.22 \%)$ & $7(20 \%)$ & 7 (13.46\%) & $0(0 \%)$ & $<0.001$ \\
\hline \multicolumn{6}{|l|}{ OS_STATUS, n (\%) } \\
\hline $0:$ LIVING & $64(46.72 \%)$ & $21(60 \%)$ & 27 (51.92\%) & $16(32 \%)$ & \\
\hline 1:DECEASED & $73(53.28 \%)$ & $14(40 \%)$ & $25(48.08 \%)$ & $34(68 \%)$ & 0.024 \\
\hline \multicolumn{6}{|l|}{ OS_DAYS } \\
\hline Mean+/-sd & $\begin{array}{l}1622.13+/- \\
1094.61\end{array}$ & $\begin{array}{l}1826.94+/- \\
1162.02\end{array}$ & $1727.88+/-1176.46$ & $1368.78+/-916.92$ & \\
\hline Median (IQR) & $1544(723,2325)$ & $2064(806,2500)$ & $\begin{array}{l}1543.5 \\
(805.75,2267.5)\end{array}$ & $\begin{array}{l}1323.5 \\
(487.25,2012.75)\end{array}$ & 0.106 \\
\hline \multicolumn{6}{|l|}{ OS_MONTHS } \\
\hline Mean+/-sd & $53.81+/-35.97$ & $60.46+/-38.14$ & $57.29+/-38.68$ & $45.54+/-30.19$ & \\
\hline Median (IQR) & $51(24,77)$ & $68(27,82.5)$ & $51(26.75,75.25)$ & $44(16.75,67)$ & 0.112 \\
\hline
\end{tabular}

* $p$-values were based on the non-parametric Kruskal-Wallis test for continuous variables and Fisher's exact test for categorical variables. 
bioRxiv preprint doi: https://doi.org/10.1101/2021.05.09.442765; this version posted May 10, 2021. The copyright holder for this preprint (which was not certified by peer review) is the author/funder. All rights reserved. No reuse allowed without permission.

\section{Fig 1}

a

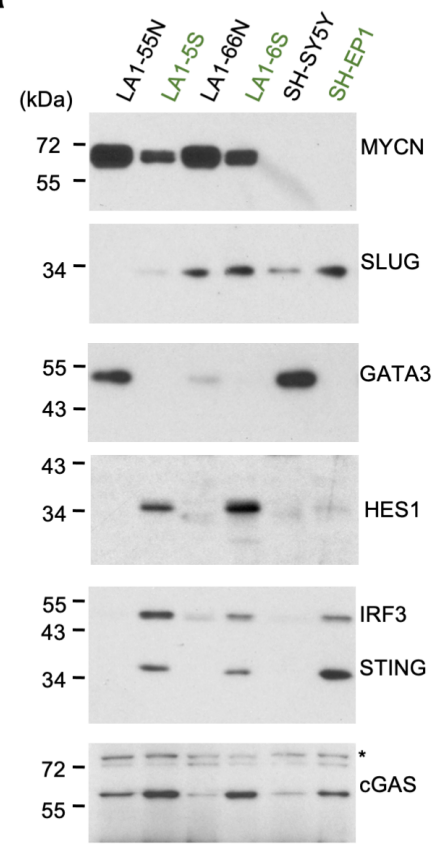

b
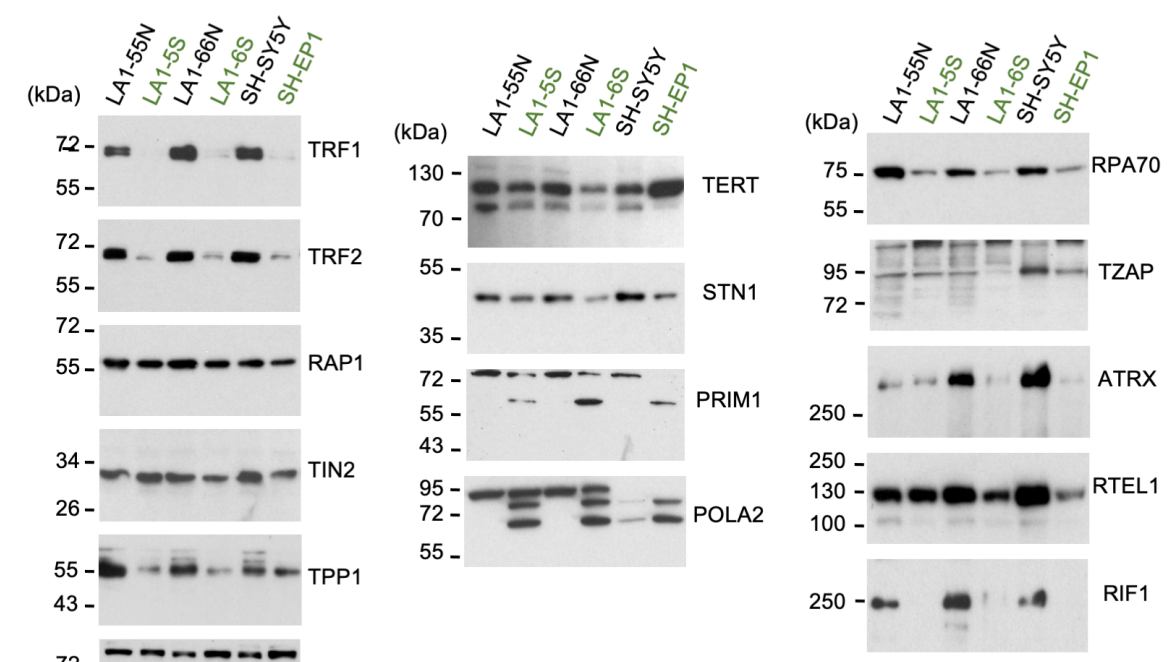

C
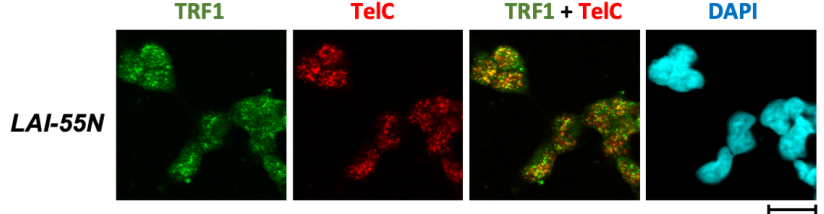

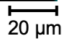
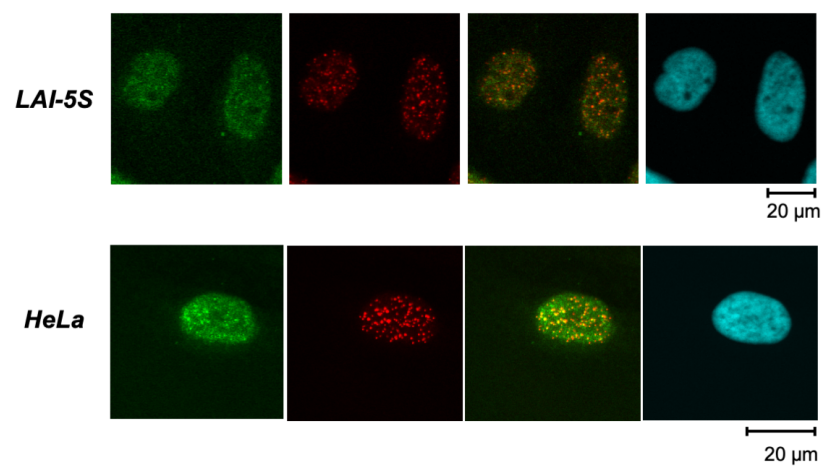

d
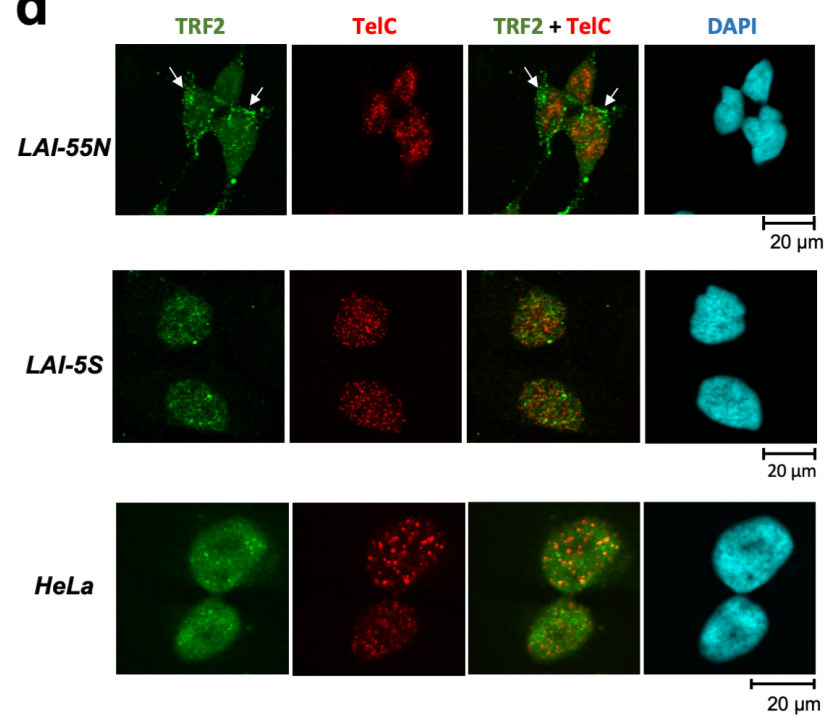

Figure 1. Matched ADRN and MES cell lines show prominent differences in telomere protein expression and localization

(a) and (b) Western analysis of cell lineage, DNA sensing, and telomere-related factors in three matched ADRN and MES cell lines. The LA1-55N (ADRN) and LA1-5S (MES) pair, as well as the LA1-66N (ADRN) and LA1-6S (MES) pair, were both derived from LAN-1, a MYCN-amplified NB tumor. The SH-SY5Y (ADRN) and SH-EP1 (MES) pair was derived from a non-MYCN-amplified tumor.

(c) and (d) IF-FISH analysis of TRF1 and TRF2 in the indicated cell lines. Prominent examples of peri-nuclear staining of TRF2 in LA1-55N are marked white arrows. 
bioRxiv preprint doi: https://doi.org/10.1101/2021.05.09.442765; this version posted May 10, 2021. The copyright holder for this preprint (which was not certified by peer review) is the author/funder. All rights reserved. No reuse allowed without permission.

\section{Fig 2}

a

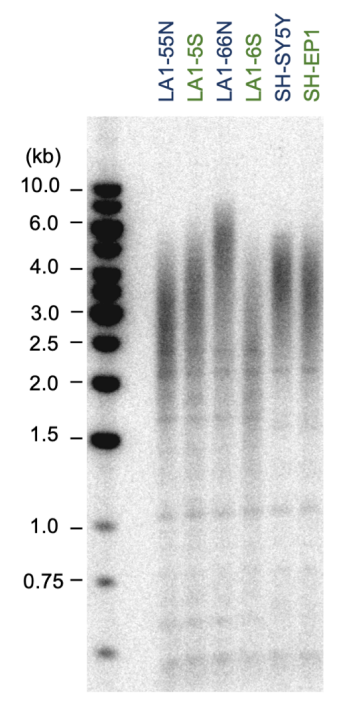

d

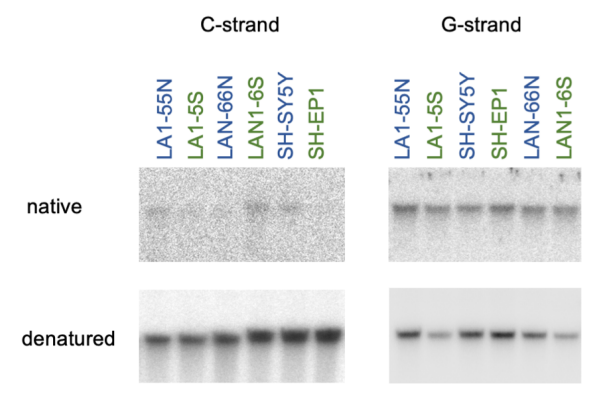

b

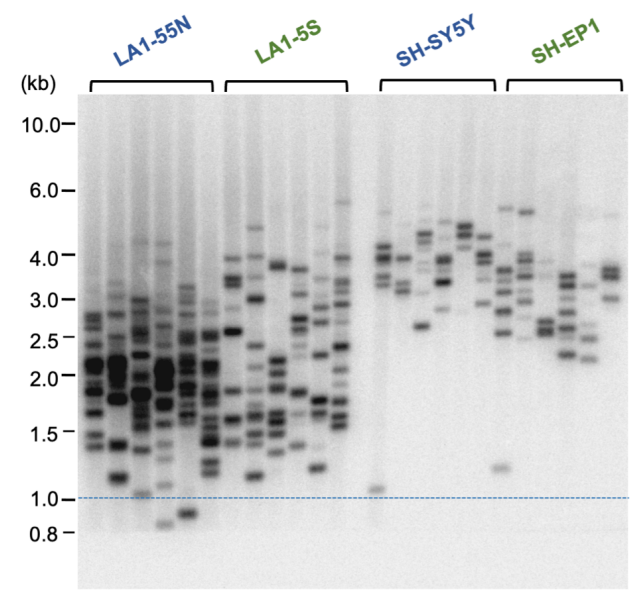

C

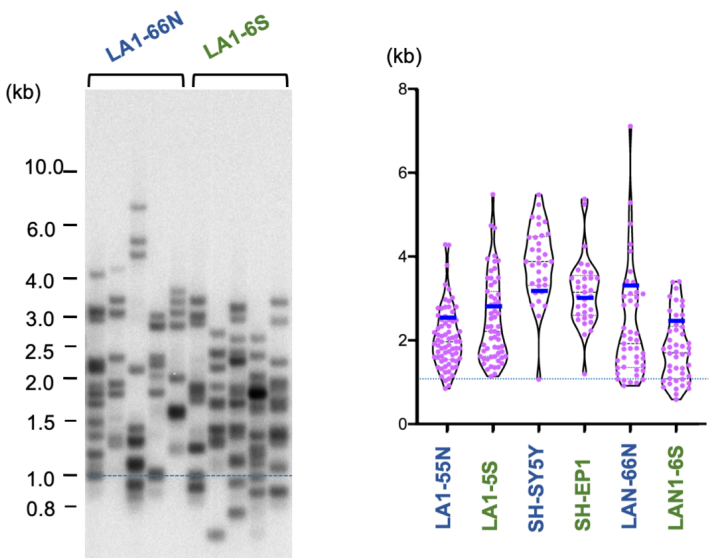

Figure 2. Characterization of telomere lengths, telomere ssDNA, and telomerase activity in matched ADRN and MES cell lines.

(a) The telomeres of the indicated cell lines were subjected to TRF Southern analysis.

(b) The telomeres of the indicated cell lines were subjected to STELA analysis. Extra-short telomeres $(<1 \mathrm{~Kb})$ are demarcated by horizontal red lines across the panels.

(c) The STELA fragments in b were analyzed using TeSLA software and the results plotted.

(d) The amount of ssDNA in the telomere G- and C-strand in the indicated cell lines were analyzed by native in-gel hybridization.

(e) TRAP analysis of telomerase activity in the indicated cell lines. 
Fig. 3

a

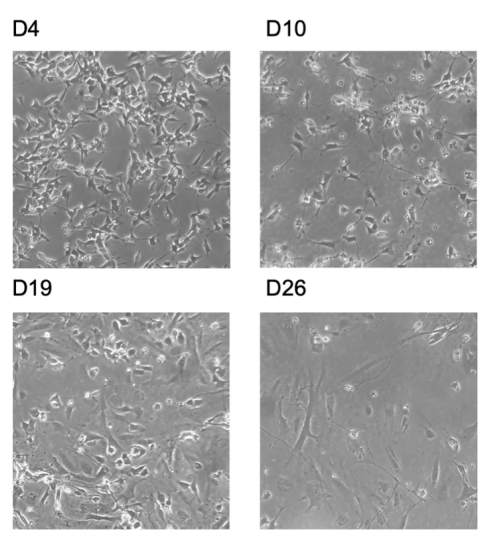

b
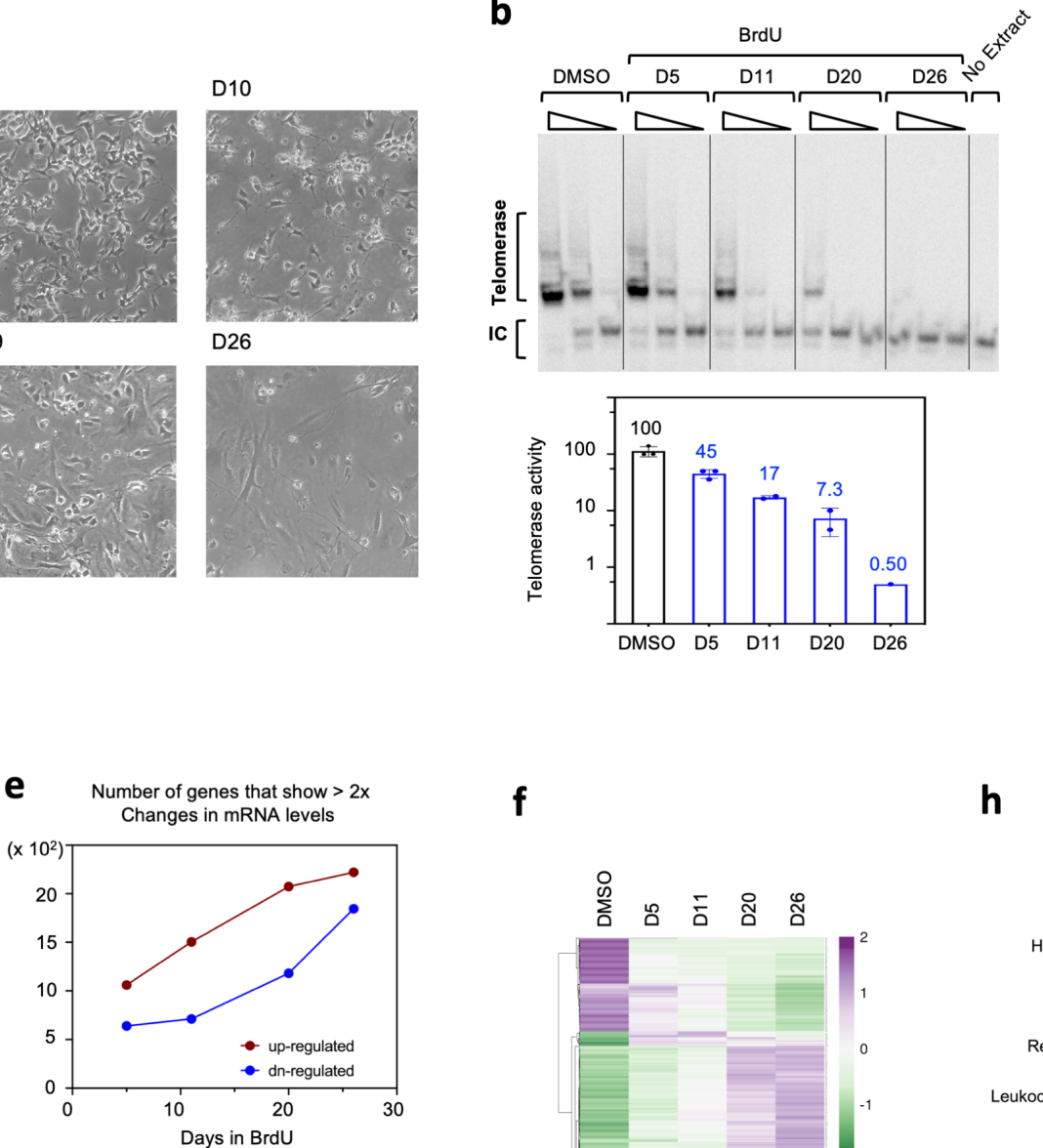

g

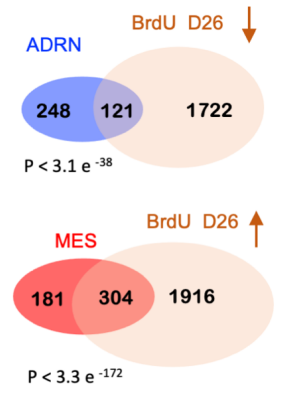

f

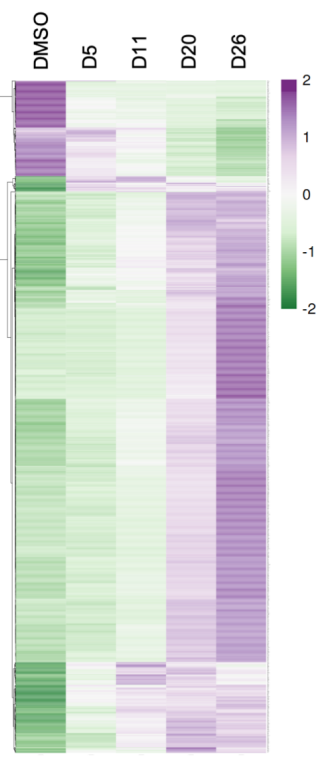

h
C

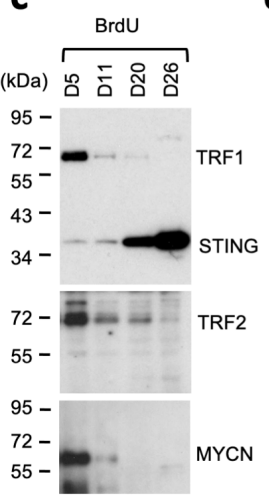

d

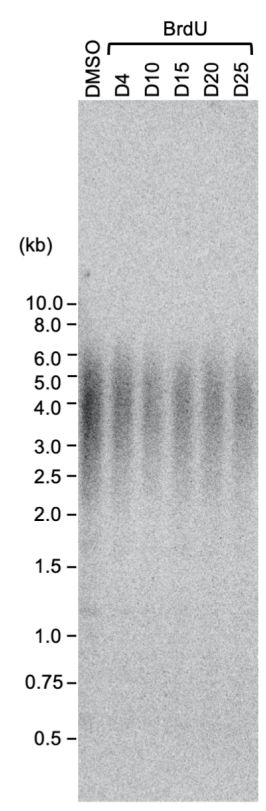

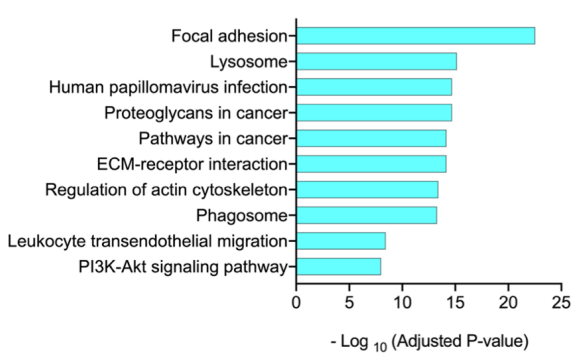

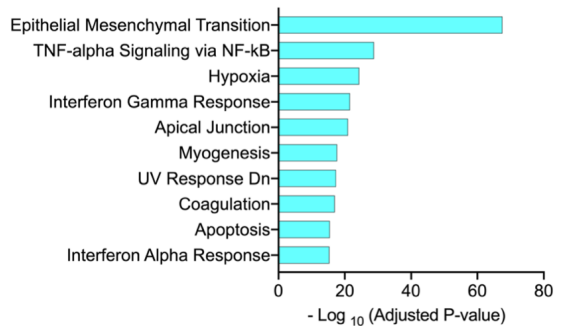

\section{Figure 3. Profiling of $B E(2) N$ cells during BrdU-induced switch from ADRN to MES cell types}

(a) Morphology of $\mathrm{BE}(2) \mathrm{N}$ at different time points following BrdU treatment. (b) TRAP analysis of telomerase activity in $\mathrm{BE}(2) \mathrm{N}$ at different time points during BrdU-induced phenotypic switch. The assays were performed using serial dilutions of the extracts (equivalent to 500,50 and $5 \mathrm{ng}$ of proteins), and the relative activities are plotted at the bottom. (c) Western analysis of telomere and lineage-related proteins in $B E(2) N$ during BrdU treatment. (d) Analysis of telomere length distributions in $\mathrm{BE}(2) \mathrm{N}$ at different time points during BrdU-induced phenotypic switch. (e) The number of up-regulated and down-regulated genes in BrdU-treated BE(2)N (by > 2-fold in comparison to the DMSO-treated control cells) were determined from RNA-seq analysis and plotted. (f) The expression patterns of the $\sim 1,100$ genes that show the greatest changes in mRNA levels ( $>3$ fold) in BrdU-treated BE(2)N were displayed using Heatmap. (g) The list of genes that were down-regulated in BrdU-treated BE(2)N (by >2-fold) was compared to the ADRN signature list ${ }^{10}$. In parallel, the list of genes that were up-regulated in BrdU-treated BE(2)N (by >2-fold) was compared to the MES signature. (h) The $\sim 2,000$ genes up-regulated in BrdU-treated $\mathrm{BE}(2) \mathrm{N}$ (by $>2$-fold) were subjected to pathway analysis by the Enrichr program. The top ten GO terms identified by the KEGG pathway database and the Hallmark gene sets in the Molecular Signature Database were ranked by adjusted $P$ value and plotted in the top and bottom panels, respectively. 
bioRxiv preprint doi: https://doi.org/10.1101/2021.05.09.442765; this version posted May 10, 2021. The copyright holder for this preprint (which was not certified by peer review) is the author/funder. All rights reserved. No reuse allowed without permission.

Fig. 4

a

Extract

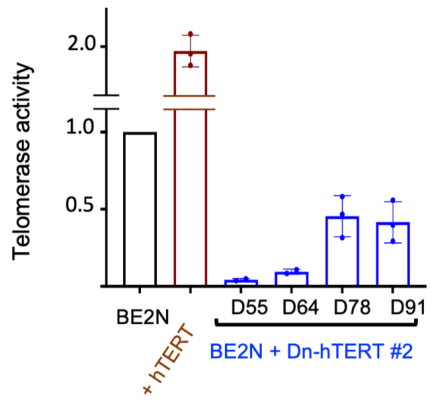

d

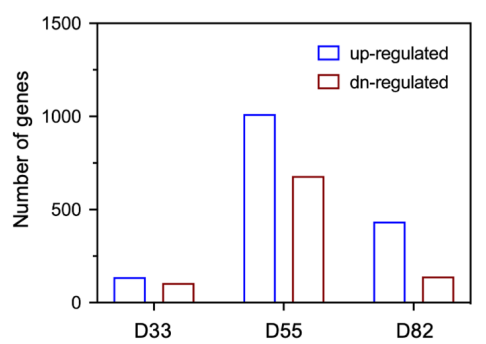

b

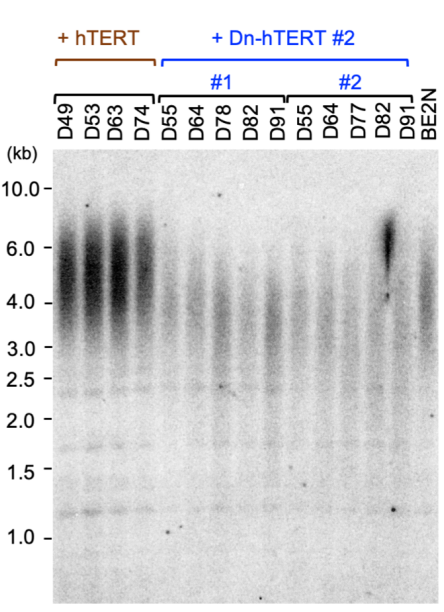

c

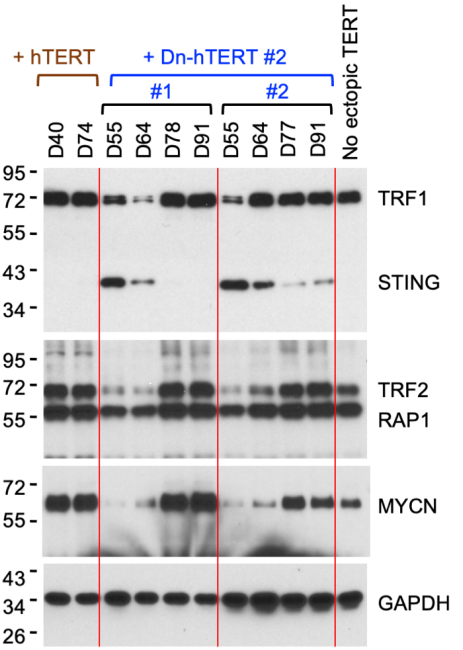

$\mathbf{e}$

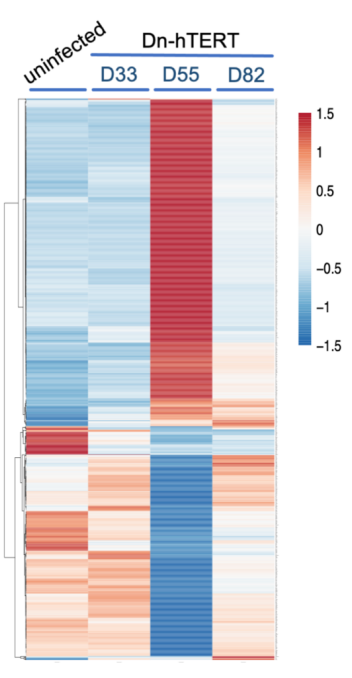

f
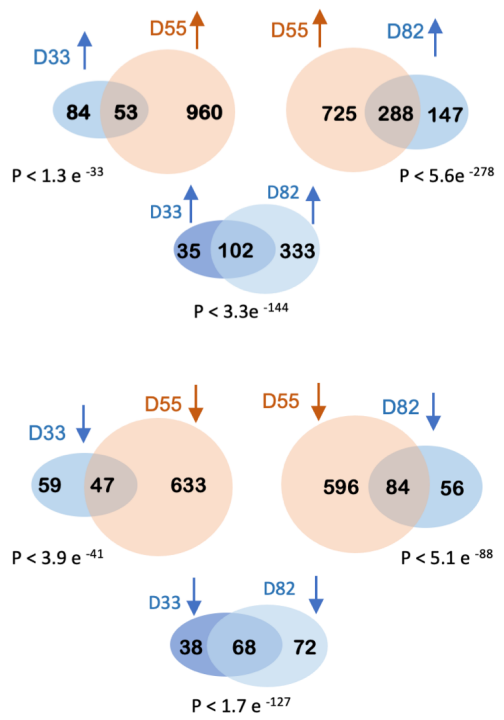

Figure 4. Profiling of BE(2)N cells during Dn-hTERT-induced switch from ADRN to MES cell types

(a) TRAP analysis of telomerase activity in BE(2)N harboring either hTERT or Dn-hTERT at different time points following retrovirus infection. The assays were performed using serial dilutions of the extracts as indicated. The relative activity of each extract was quantified using ImageQuant and plotted at the bottom.

(b) Analysis of telomere length distributions in $\mathrm{BE}(2) \mathrm{N}$ harboring either hTERT or Dn-hTERT at different time points following retrovirus infection.

(c) Western analysis of telomere and lineage-related proteins in BE(2)N during passage of cells infected with viruses that harbor either hTERT or DnhTERT.

(d) The number of up-regulated and down-regulated genes in Dn-hTERT-treated BE(2)N at the indicated time points (by > 2-fold in comparison to the DMSO-treated control cells) were determined from RNA-seq analysis and plotted.

(e) The expression patterns of the $\sim 1,000$ genes that show the greatest changes in mRNA levels ( $>2$ fold) in BrdU-treated BE(2)N were displayed using Heatmap.

(f) (top) Pair-wise comparison of the degree of overlaps between genes up-regulated by Dn-hTERT treatment at different time points. (bottom) Pair-wise comparison of the degree of overlaps between genes down-regulated by Dn-hTERT treatment at different time points. 
bioRxiv preprint doi: https://doi.org/10.1101/2021.05.09.442765; this version posted May 10, 2021. The copyright holder for this preprint (which was not certified by peer review) is the author/funder. All rights reserved. No reuse allowed without permission.

Fig. 5
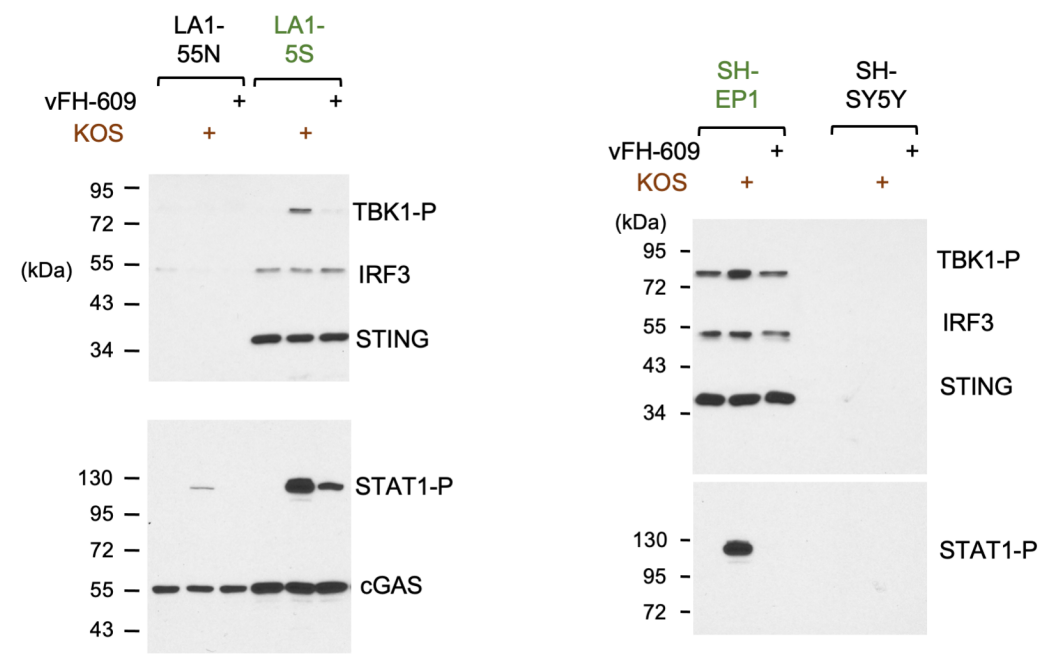

Figure 5. Activation of the DNA sensing pathway in ADRN and MES cells by Herpes virus

(a) Western analysis of factors in the DNA sensing pathway in LA1-55N and LA1-5S cell lines following Herpes virus infection.

(b) Western analysis of factors in the DNA sensing pathway in SH-SY5Y and SH-EP1 cell lines following Herpes virus infection. 
bioRxiv preprint doi: https://doi.org/10.1101/2021.05.09.442765; this version posted May 10, 2021. The copyright holder for this preprint (which was not certified by peer review) is the author/funder. All rights reserved. No reuse allowed without permission.

\section{Fig. 6}

a

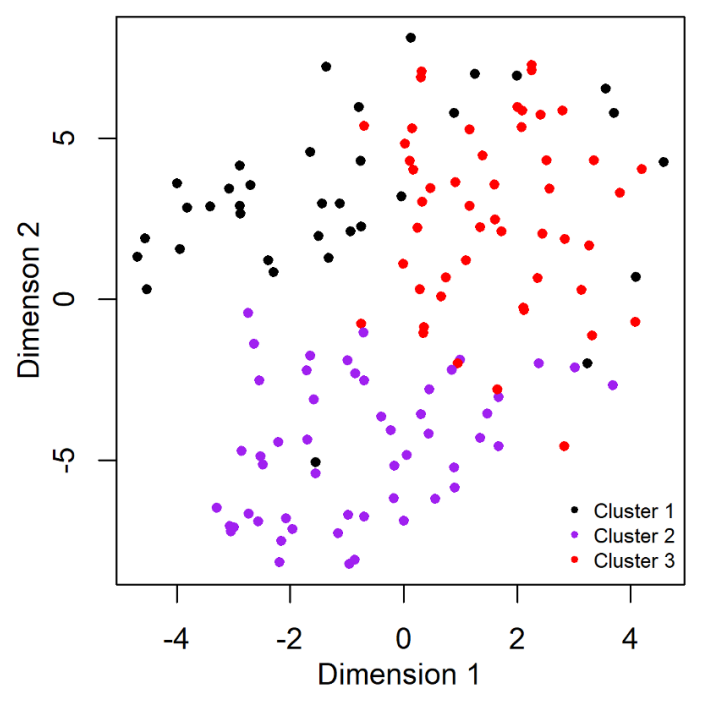

b

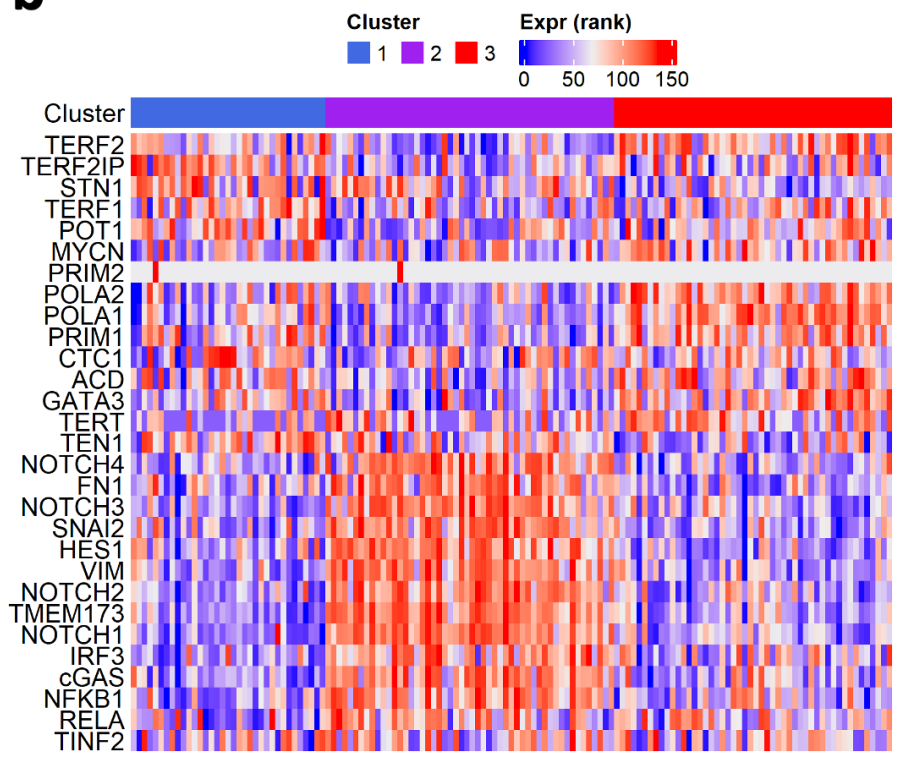

C

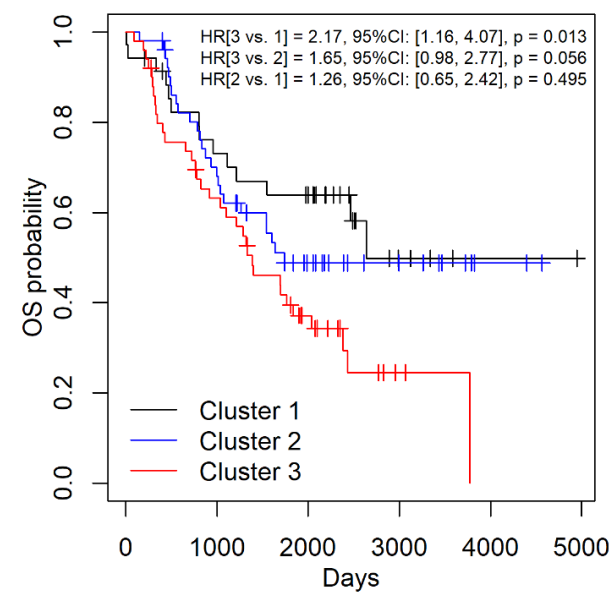

d

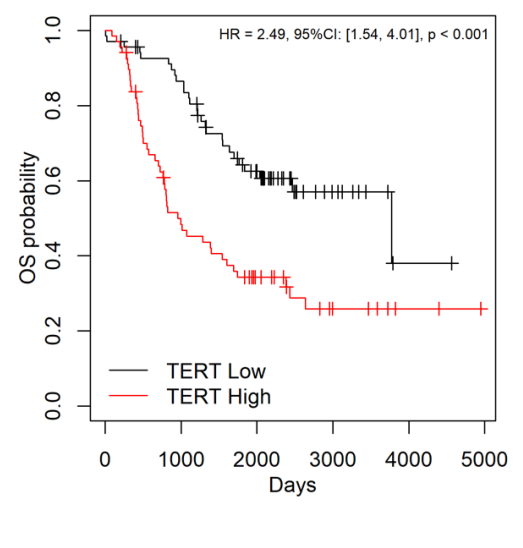

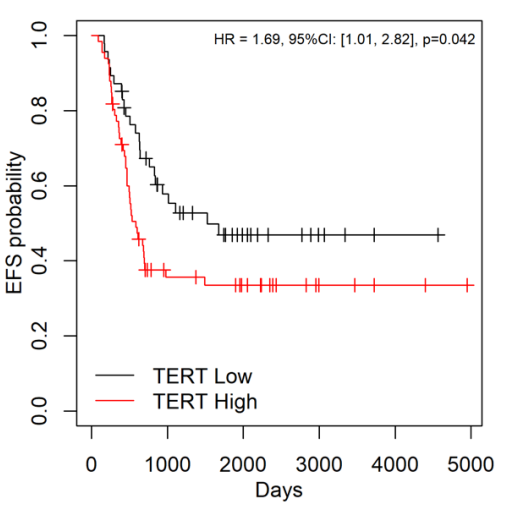

Figure 6. Analysis of clinical tumor samples using a telomere- and cell lineage-related signature gene list

(a) The RNA levels (rank transformed) of a list of 29 telomere-, cell lineage- and immunity-related genes across 137 NB tumors with RNA-seq data were analyzed and clustered using PAM and displayed as a t-SNE plot.

(b) The rank transformed RNA levels of signature genes across clustered NB tumor samples are displayed in Heatmap.

(c) Kaplan-Meier overall survival curves for three clusters of patients with distinct gene expression profiles.

(d) Kaplan-Meier overall survival and event free survival curves for patients with high TERT and low TERT expression. 